\title{
Synthesis of substituted naphthalenes from a-tetralones generated by a xanthate radical addition-cyclisation sequence
}

\author{
Alejandro Cordero-Vargas, Inés Pérez-Martín, Béatrice Quiclet-Sire and Samir Z. Zard* \\ Laboratoire de Synthèse Organique associé au CNRS, Ecole Polytechnique, 91128 Palaiseau, \\ France.E-mail: zard@poly.polytechnique.fr
}

Received 23rd July 2004, Accepted 1st September 2004

First published as an Advance Article on the web 27th September 2004

A simple, highly efficient and cheap synthesis of substituted naphthalenes is reported. These aromatic compounds can be easily prepared in acidic or basic conditions from a-tetralones, obtained by a xanthate-mediated additioncyclisation sequence.

\section{Introduction}

Much attention has recently been focused on the regioselective synthesis of substituted naphthalene derivatives. ${ }^{1}$ These compounds constitute the basic skeleton of many biologically important natural products and pharmaceuticals ${ }^{2}$ and synthetic routes to the naphthalene moiety are highly desirable.

Due to their stability, these aromatic compounds react with difficulty under mild conditions and, on many occasions, the use of drastic methods results in non-regioselective reactions. In addition, when regioselectivity exists, it usually depends on the nature of the substituents already present in the aromatic ring (electron-withdrawing or electron-donating groups).

In order to circumvent these limitations, we have developed a simple methodology for the preparation of the naphthalene skeleton by using the functionalisation of an a-tetralone and its aromatisation under acidic or basic conditions, independently of the nature of the substituents in the ring (Scheme 1).<smiles>[R]C=Cc1ccc2c(c1[R])C([R])CCC(=O)C2CC</smiles>

Scheme 1

It is known that tetralones can be transformed into their corresponding aromatic structures by different methods such as oxidation with DDQ or $\mathrm{Pd} / \mathrm{C}$ at high temperatures. However, commercially available tetralones are limited, as well as general methods to obtain functionalised precursors for the desired aromatic systems.

\section{Results and discussion}

A few years ago, we reported a new method for the preparation of a-tetralones using xanthate free radical chemistry (Scheme 2). ${ }^{3}$<smiles></smiles>

$1 \mathrm{Xa}=$ SCSOEt

$$
\underset{\mathrm{DLP}}{\stackrel{\mathrm{2}_{2}}{\longrightarrow}}
$$<smiles>[R]CCCC(=O)c1ccc([R])cc1</smiles>
DLP<smiles>[R]C=Cc1ccccc1C([R2])C</smiles>
4
Scheme 2 Radical addition-cyclisation sequence.

We had shown that xanthates such as $\mathbf{1}$ undergo a radical chain reaction to a great variety of olefinic traps $\mathbf{2}$ to give adduct $\mathbf{3}$, which can be used as a starting point for another radical sequence, in this case, to construct the six-membered ring of a-tetralone 4. This methodology has important virtues such as simplicity, cheapness, absence of heavy metals, ease of scale-up and the possibility of operating under fairly concentrated conditions.

With the aim of accessing substituted naphthalenes, obtained from modified a-tetralones, we prepared different precursors through a two-step one-pot protocol using a range of xanthates and olefins (Table 1). This is in contrast to our earlier work where the intermediate adduct $\mathbf{3}$ was isolated.

The selection of vinyl pivalate $\mathbf{2} \mathbf{a}$ as the radical trap was not arbitrary. It was anticipated that the OPiv group could serve as a leaving group for the aromatisation in acidic media in order to facilitate the last step of the route. Furthermore, the pivaloxy group is compatible with a number of useful transformations as shown below.

One important aspect that emerges from inspection of the examples in Table 1 is that in principle, practically all of the positions of the tetralone structure may be functionalised by the appropriate choice of the initial xanthate and olefin components or by subsequent modification of the product. Although the overall yields of tetralones $\mathbf{4 a - 4 f}$ may seem moderate, it must be realised that they correspond to two difficult radical processes: an intermolecular addition to a non-activated olefin and a ring closure to an aromatic ring. Both of these steps would have been difficult to perform using other radical methods. As for substituents on the starting xanthate moiety, both electrodonating and electron-withdrawing groups are tolerated.

In the case of tetralones $\mathbf{4 a}, \mathbf{4 b}$ and $\mathbf{4 c}$, we found that addition of camphorsulfonic acid (CSA) during the ring-closing step was beneficial and allowed up to $20 \%$ increase in yield. Protonation of the ketone oxygen presumably causes a speeding up of the radical addition to the aromatic ring. ${ }^{4}$ In contrast, with $\mathbf{4 d}$ and 4f, which contain electron donating groups, the addition of CSA proved deleterious causing a rapid degradation and a dramatic lowering of the yield (to about 10\%). The reason may be the premature elimination of the pivaloxy group. Such an elimination is facilitated by electron releasing substituents.

Preparation of the required naphthalenes was accomplished starting from these suitably substituted tetralones or by modification of the precursors followed by aromatisation under acidic or basic conditions as summarized in Table 2 and Scheme 3.

The initial experiments were carried out directly on the tetralones $\mathbf{4 a}$, 4e and $\mathbf{4 f}$ (entries 1, 2 and 3) employing $p$-toluenesulfonic acid (PTSA) in refluxing toluene. We obtained the corresponding naphthols in good yields, except in the case of tetralone $\mathbf{4 f}$ (entry 3 ), where a complex mixture of products was observed.

Next, a simple, but important functionalisation was made prior to the aromatisation step. In this way, tetralones $\mathbf{4 a}, \mathbf{4 c}$, $\mathbf{4 d}$ and $\mathbf{4 f}$ were treated with pyridinium bromide perbromide, to give compounds 7, 9, 12 and $\mathbf{1 4}$ respectively, without affecting the pivalate group. ${ }^{5}$ These compounds were then subjected directly to different aromatisation processes. Thus, in the case 
Table 1 Formation of a-tetralones employed like precursors ${ }^{a}$

Olefin $\mathbf{2}$

of 7 and 9 the reaction was carried out under acidic conditions with PTSA and pyridinium bromide perbromide, respectively, and the corresponding a-bromonaphthols were produced in good yields (entries 4 and 5). These compounds are important in organic synthesis because they are substrates for various transition metal catalysed reactions and are appropriate precursors for the generation of benzynes which, although not isolable, act as dienophiles that can be trapped with dienes in Diels-Alder reactions to give more complex tricyclic compounds. ${ }^{6}$ Alternatively, the treatment of tetralones 7 and 12 with $\mathrm{Li}_{2} \mathrm{CO}_{3}$ and $\mathrm{LiBr}$ in dimethylformamide gave regioselectively monoprotected naphthalenediols 11 and 13 in 57 and 64\% yield respectively (entries 6 and 7), but unfortunately, in the case of the tetralone 14, a complex mixture was obtained (entry 8 ).

Tetralone 15, which possesses a xanthate group at the carbon in the a position to the carbonyl group, was obtained in $68 \%$ yield by nucleophilic displacement of the bromine atom in tetralone 7 . The aromatisation was carried out in the presence of PTSA, and the product $\mathbf{1 6}$ was obtained in excellent yield, resulting from the aromatisation process and nucleophilic addition of the resulting phenolic oxygen onto the thiocarbonyl group (entry 9).

When a-bromotetralone 7 was treated with ethyl cyanoacetate and $\mathrm{K}_{2} \mathrm{CO}_{3}$, naphthalene 17 was produced in $51 \%$ yield after acidification with an aqueous citric acid solution (entry 10). ${ }^{7}$

In the formation of naphthol 18, advantage was taken of the nucleophilic character of the a-carbon to the carbonyl group in the corresponding tetralone to carry out an aldol condensation with benzaldehyde. The isomerisation of the exocyclic double bond in the basic medium generates the corresponding naphthol 18 in moderate yield (entry 11).
These last two examples illustrate the possibility of creating $\mathrm{C}-\mathrm{C}$ bonds next to the carbonyl group in order to obtain more complex naphthalene structures. In recent times, the synthesis of such structures has relied heavily on transition metal catalysed processes. ${ }^{8}$

Naphthylamines can be obtained via the corresponding Schiff base. For example, when compound $\mathbf{4 b}$ was treated with benzylamine followed by in situ aromatisation of the intermediate imine with $\mathrm{AlCl}_{3}$, naphthalene 19a was produced in good yield (entry 12). When a Fischer indole synthesis was carried out with the same tetralone $\mathbf{4 b}$, by treatment with phenylhydrazine in polyphosphoric acid, ${ }^{9}$ the tetracyclic compound $\mathbf{2 0}$ was obtained in $56 \%$ yield (entry 13). These types of products have attracted a great deal of attention from synthetic as well as medicinal chemists because such structures are present in a number of natural products. ${ }^{10}$

Finally, examples of treatment of the a-tetralones with carbon nucleophiles and aromatisation are depicted in Scheme 3.

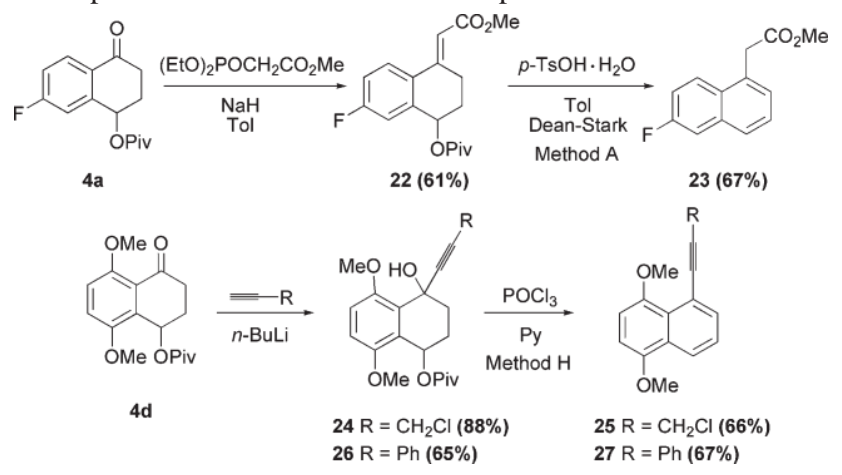

Scheme 3 Aromatisation of compounds derived from tetralones. ${ }^{11}$

In the first case, starting from the tetralone $\mathbf{4 a}$ and applying a Horner-Emmons reaction, olefin $\mathbf{2 2}$ was obtained in good yield. The aromatisation process was carried out with $p$ toluenesulfonic acid to produce naphthalene $\mathbf{2 3}$ in $67 \%$ yield.

Lithium acetylides could also be added to the tetralone carbonyl without affecting the pivaloxy group as illustrated by the efficient synthesis of $\mathbf{2 4}$ and 26. Subsequent aromatization with $\mathrm{POCl}_{3} / \mathrm{Py}$ gave the corresponding naphthalenes. In these cases, the usual treatment with PTSA was not satisfactory. Compound $\mathbf{2 5}$ is especially interesting since it contains a reactive propargyl chloride and thus represents a springboard for numerous subsequent transformations.

In summary, we have described a convergent approach to naphthalene structures with a diverse pattern of substitution. The process involves cheap, readily available reagents and is based on radical chemistry that does not involve the use of heavy metals such as tin or mercury.

\section{Experimental}

All reactions were carried out under an inert atmosphere. Commercial reagents were used as received without further purification. All products were purified by using silica gel SDS $60 \mathrm{C}$. C. 40-63 or by crystallisation. NMR spectra were recorded in $\mathrm{CDCl}_{3}$ with TMS as an internal standard at room temperature on a Bruker AMX400 operating at $400 \mathrm{MHz}$ for ${ }^{1} \mathrm{H}$ and $100 \mathrm{MHz}$ for ${ }^{13} \mathrm{C}$. Infrared absorption spectra were recorded as a solution in $\mathrm{CCl}_{4}$ with a Perkin-Elmer 1600 Fourier Transform Spectrophotometer. Some mass spectra were determined at 70 $\mathrm{eV}$ with an AutoSpec Micromass and the others were recorded with an HP 5989B mass spectrometer using ammonia as the reagent gas. Melting points were determined using a Reichert microscope apparatus and were uncorrected.

Dithiocarbonic acid [2-(2,5-dimethoxy-phenyl)-2-oxo-ethyl] ester ethyl ester (1c)

To a cold $\left(0{ }^{\circ} \mathrm{C}\right)$ solution of 2,5-dimethoxy-2 $\square$-bromoacetophenone ${ }^{12}(5 \mathrm{~g}, 19.29 \mathrm{mmol})$ in acetone $(38.6 \mathrm{~mL})$ 
Table 2 Aromatisation of substituted a-tetralones

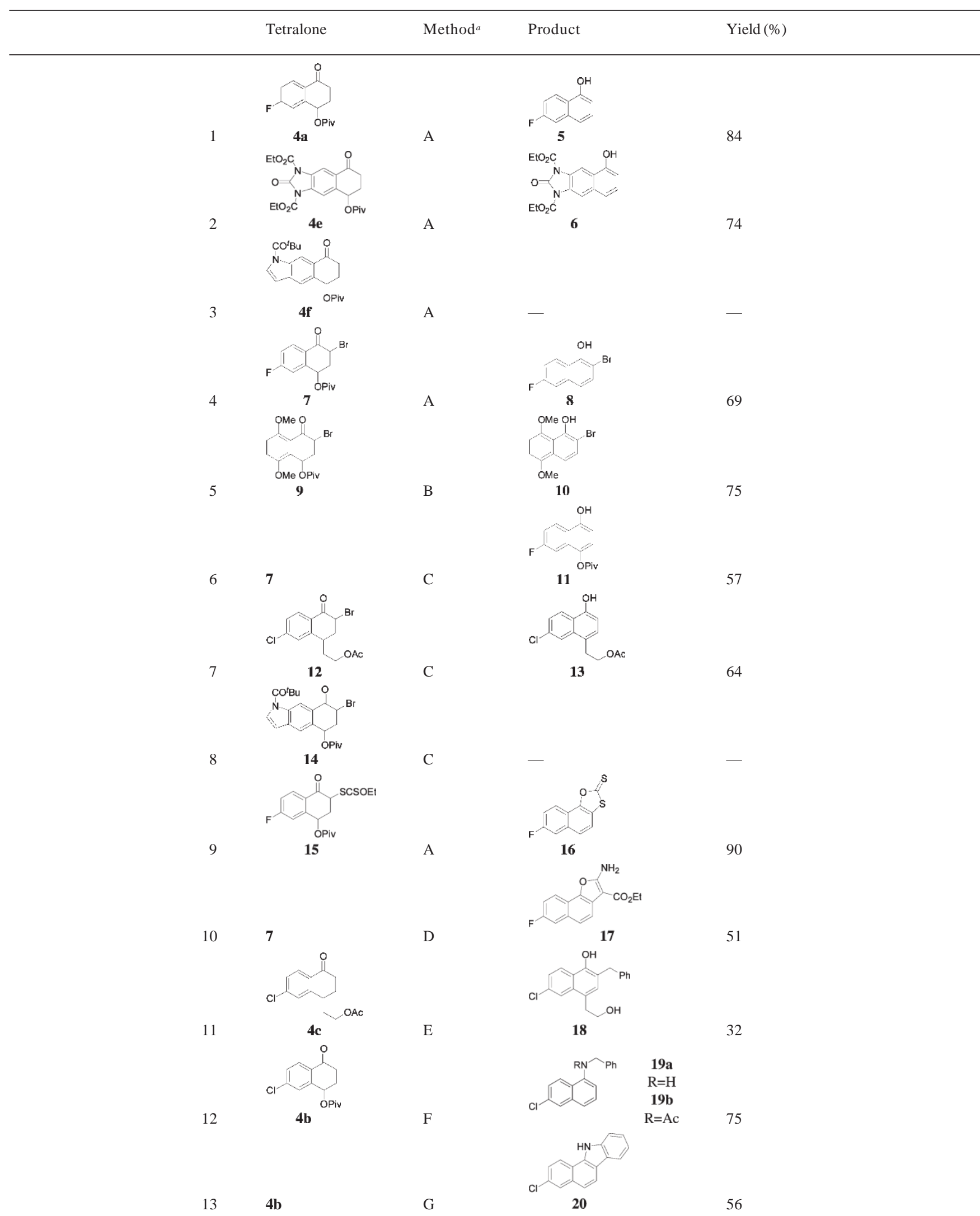

${ }^{a}$ METHOD A: Tetralone derivative $(1 \mathrm{mmol})$ in toluene $(35 \mathrm{~mL})$ containing $p$-toluenesulfonic acid $(3 \mathrm{mmol})$ was refluxed using a Dean-Stark apparatus. METHOD B: Tetralone derivative $(1 \mathrm{mmol})$ in acetic acid $(10 \mathrm{~mL})$ containing pyridinium bromide perbromide $(1 \mathrm{mmol})$ was stirred at room temperature for $1 \mathrm{~h}$. METHOD C: Tetralone derivative $(1 \mathrm{mmol})$ in dry dimethylformamide $(6 \mathrm{~mL}) \mathrm{containing} \mathrm{Li}_{2} \mathrm{CO}_{3}(2 \mathrm{mmol})$ and $\mathrm{LiBr}_{(2}$ mmol) was warmed at $140{ }^{\circ} \mathrm{C}$. METHOD D: To a cold solution of ethyl cyanoacetate $(12 \mathrm{mmol})$ in acetone $(2 \mathrm{~mL})$ was added $\mathrm{K}_{2} \mathrm{CO}{ }_{3}(3 \mathrm{mmol})$ and the tetralone $(1 \mathrm{mmol})$ was added at $0{ }^{\circ} \mathrm{C}$. The mixture was stirred at room temperature and then acidified with an aqueous solution of citric acid. METHOD E: To the tetralone derivative $(1 \mathrm{mmol})$ and benzaldehyde $(1.5 \mathrm{mmol})$ in dry tert-butanol $(10 \mathrm{~mL}) \mathrm{was}$ added $t$-BuOK $(2 \mathrm{mmol})$ and the mixture was heated under reflux conditions. METHOD F: Tetralone derivative $(1 \mathrm{mmol})$ in dry toluene $(10 \mathrm{~mL})$ containing benzylamine $(2 \mathrm{mmol})$ was heated under reflux for $3 \mathrm{~h}$. Then, $\mathrm{AlCl}_{3}(2 \mathrm{mmol})$ was added at room temperature and the solution was refluxed for $15 \mathrm{~min}$. METHOD G: Fischer indole synthesis. The tetralone $(1 \mathrm{mmol})$ was treated with phenylhydrazine $(2 \mathrm{mmol})$ and polyphosphoric acid $(2.5 \mathrm{mmol})$, and the mixture was heated at $100{ }^{\circ} \mathrm{C}$ for $15 \mathrm{~min}$. 
was added portionwise potassium $O$-ethylxanthate $(3.4 \mathrm{~g}$,

$21.22 \mathrm{mmol}$ ). The reaction mixture was stirred at $0{ }^{\circ} \mathrm{C}$ for a further $1 \mathrm{~h}$, the solvent was evaporated and the resulting mixture partitioned between water and $\mathrm{CH}_{2} \mathrm{Cl}_{2}$. The aqueous phase was extracted with $\mathrm{CH}_{2} \mathrm{Cl}_{2}$, the combined organic extracts were dried over $\mathrm{Na}_{2} \mathrm{SO}_{4}$ and concentrated under reduced pressure. Recrystallization of the residue from $\mathrm{CH}_{2} \mathrm{Cl}_{2}$-petroleum ether gave xanthate $1 \mathrm{c}\left(95 \%\right.$ yield) as yellow crystals. $\mathrm{mp}=71-72{ }^{\circ} \mathrm{C}$; $m_{\max } / \mathrm{cm}^{-1} 1676\left(\mathrm{C}\right.$ 回O), $1223(\mathrm{~S}-C$ 回 $), 1050(S-C$ 回 $) ; d_{\mathrm{H}}(400$ MHz) $7.31(1 \mathrm{H}, \mathrm{d}, J=3.2 \mathrm{~Hz}, \mathrm{C} H$ arom $), 7.07(2 \mathrm{H}, \mathrm{dd}, J=$ 9.2, $3.2 \mathrm{~Hz} \mathrm{CH}$ arom), $6.93(1 \mathrm{H}, \mathrm{d}, J=9.2 \mathrm{~Hz}, \mathrm{CH}$ arom), 4.59-4.64 (4H, m, $\mathrm{OCH}_{2}$ and $\left.\mathrm{CH}_{2}-\mathrm{S}\right), 3.91\left(3 \mathrm{H}, \mathrm{s}, \mathrm{OCH}_{3}\right), 3.78$ $\left(3 \mathrm{H}, \mathrm{s}, \mathrm{OCH}_{3}\right), 1.39\left(3 \mathrm{H}, \mathrm{dd}, J=7.2,7.2 \mathrm{~Hz}, \mathrm{CH}_{3}\right) ; d_{\mathrm{C}}(100$ $\mathrm{MHz}) 213.8(\mathrm{C}, C$ 回 $), 193.5$ (C, C回O), 153.8 (C, C-OMe), $153.4(\mathrm{C}, C-\mathrm{OMe}), 126.6(\mathrm{C}, C-\mathrm{CO}), 121.3(\mathrm{CH}, C \mathrm{CH}$ arom), $114.4(\mathrm{CH}, \mathrm{CH}$ arom $), 113.2(\mathrm{CH}, \mathrm{CH}$ arom $), 70.3\left(\mathrm{CH}_{2}\right.$, $\left.\mathrm{OCH}_{2}\right), 56.2\left(\mathrm{CH}_{3}, \mathrm{OCH}_{3}\right), 55.9\left(\mathrm{CH}_{3}, \mathrm{OCH}_{3}\right), 47.7\left(\mathrm{CH}_{2}\right.$, $\left.\mathrm{CH}_{2}-\mathrm{S}\right), 13.8\left(\mathrm{CH}_{3}\right) ; \mathrm{m} / \mathrm{z}\left(\mathrm{CI}+\mathrm{NH}_{3}\right) 318\left(\mathrm{MH}^{+}+\mathrm{NH}_{3}\right), 301$ (Aenteral procedure for the preparation of tetralones $4 \mathrm{a}-4 \mathrm{f}$

A solution of xanthate $(1 \mathrm{mmol})$ and vinyl pivalate $(2 \mathrm{mmol})$ in 1,2-dichloroethane (DCE) $(1 \mathrm{~mL})$ was refluxed for 15 min under argon. Lauroyl peroxide (DLP) (5 mol\%) was then added to the refluxing solution, followed by additional portions $(2.5 \mathrm{~mol} \%$ every $90 \mathrm{~min}$ ). When starting material was completely consumed the mixture was cooled to room temperature, concentrated under reduced pressure and the crude mixture, redissolved in 1,2-dichloroethane $(10 \mathrm{~mL})$ together with camphorsulfonic acid (0.1 mmol, when electron-withdrawing groups are present in the aromatic ring). DLP was then added to the refluxing solution (20 mol\% every hour). When starting material was totally consumed, the mixture was cooled to room temperature, concentrated under reduced pressure and purified by flash column chromatography (silica gel, petroleum ether-ethyl acetate, a small layer of basic alumina was placed on the top of the silica to remove any lauric acid present) to give the final tetralone.

7-Fluoro-4-oxo-1,2,3,4-tetrahydro-1-naphthalenyl pivalate (4a)

Obtained from xanthate $1 \mathbf{a}^{13}$ as a yellow oil (49\%) (silica gel, petroleum ether-ethyl acetate, $9: 1) ; m_{\max } / \mathrm{cm}^{-1} 1732(\mathrm{O}-\mathrm{C}$ ? $O$ ), $1694(\mathrm{C}$ ? O $), 1148(O-C$ 回 $) ; d_{\mathrm{H}}(400 \mathrm{MHz}) 7.97(1 \mathrm{H}, \mathrm{d}$ $J=8.8,5.2 \mathrm{~Hz}, \mathrm{C} H$ arom $), 7.12(2 \mathrm{H}, \mathrm{m}, \mathrm{CH}$ arom $), 6.06(1 \mathrm{H}, \mathrm{dd}$, $J=8.0,4.0 \mathrm{~Hz}, 1-\mathrm{H}), 2.89\left(1 \mathrm{H}, \mathrm{ddd}, J=20.0,8.0,4.0 \mathrm{~Hz}, 3-\mathrm{H}_{\mathrm{a}}\right)$, $2.68\left(1 \mathrm{H}, \mathrm{ddd}, J=16.8,8.0,4.0 \mathrm{~Hz}, 3-\mathrm{H}_{\mathrm{b}}\right), 2.42\left(1 \mathrm{H}, \mathrm{m}, 2-\mathrm{H}_{\mathrm{a}}\right)$, $2.24\left(1 \mathrm{H}, \mathrm{m}, 2-\mathrm{H}_{\mathrm{b}}\right), 1.25(9 \mathrm{H}, \mathrm{s}, 3 \times \mathrm{Me}) ; d_{\mathrm{C}}(100 \mathrm{MHz}) 195.4(\mathrm{C}$, C-4), $176.3(\mathrm{C}, \mathrm{O}-\mathrm{CO}), 166.0\left(\mathrm{C}, \mathrm{C}-7,{ }^{1} J_{\mathrm{C}-\mathrm{F}}=254.6 \mathrm{~Hz}\right), 144.5$ $(\mathrm{C}, C-\mathrm{CO}), 130.6\left(\mathrm{CH}, \mathrm{d},{ }^{3} J_{\mathrm{C}-\mathrm{F}}=10.5 \mathrm{~Hz}, \mathrm{CH}\right.$ arom $), 116.4(\mathrm{CH}$, $\mathrm{d},{ }^{2} J_{\mathrm{C}-\mathrm{F}}=23.0 \mathrm{~Hz}, C \mathrm{H}$ arom $), 115.5(\mathrm{C}, C-\mathrm{CH}), 114.4(\mathrm{CH}, \mathrm{d}$, ${ }^{2} J_{\mathrm{C}-\mathrm{F}}=23.0 \mathrm{~Hz}, \mathrm{CH}$ arom $), 68.6(\mathrm{CH}, \mathrm{C}-1), 39.1\left(\mathrm{C},{ }^{t} \mathrm{Bu}\right), 34.7$ $\left(\mathrm{CH}_{2}, \mathrm{C}-3\right), 28.7\left(\mathrm{CH}_{2}, \mathrm{C}-2\right), 27.1\left(3 \times \mathrm{CH}_{3},{ }^{t} \mathrm{Bu}\right) ; \mathrm{m} / \mathrm{z}\left(\mathrm{CI}+\mathrm{NH}_{3}\right)$ $282\left(\mathrm{MH}^{+}+\mathrm{NH}_{3}\right), 265\left(\mathrm{MH}^{+}\right), 164\left(\mathrm{MH}^{+}-\mathrm{OPiv}\right) ;$ Anal. calcd. for $\mathrm{C}_{15} \mathrm{H}_{17} \mathrm{O}_{3} \mathrm{~F}$ : C, 68.17; H, 6.48. Found: C, 68.31; H, 6.65\%.

\section{7-Chloro-4-oxo-1,2,3,4-tetrahydro-1-naphthalenyl pivalate (4b)}

Obtained from xanthate $\mathbf{1 b}^{13}$ as a yellow solid (81\%), (silica gel, petroleum ether-ethyl acetate, $9: 1$ ), $\mathrm{mp} 76-80^{\circ} \mathrm{C}$ (petroleum ether); $m_{\max } / \mathrm{cm}^{-1} 1733(\mathrm{O}-C$ 回 $O), 1696(\mathrm{C}$ 回 $), 1143\left(O-\mathrm{O}_{0}, d_{\mathrm{H}}\right.$ $(400 \mathrm{MHz}) 8.00(1 \mathrm{H}, \mathrm{d}, J=8.0 \mathrm{~Hz}, \mathrm{CH}$ arom $), 7.42(1 \mathrm{H}, \mathrm{d}$, $J=8.0 \mathrm{~Hz}, \mathrm{CH}$ arom), $7.41(1 \mathrm{H}, \mathrm{s}, \mathrm{C} H$ arom $), 6.05(1 \mathrm{H}, \mathrm{dd}$, $J=8.0,4.0 \mathrm{~Hz}, 1-\mathrm{H}), 2.90(1 \mathrm{H}, \mathrm{ddd}, J=18.0,10.0,4.0 \mathrm{~Hz}, 3-$ $\left.\mathrm{H}_{\mathrm{a}}\right), 2.69\left(1 \mathrm{H}, \mathrm{ddd}, J=20.0,8.0,4.0 \mathrm{~Hz}, 3-\mathrm{H}_{\mathrm{b}}\right), 2.41(1 \mathrm{H}, \mathrm{m}$, $\left.2-\mathrm{H}_{\mathrm{a}}\right), 2.26\left(1 \mathrm{H}, \mathrm{m}, 2-\mathrm{H}_{\mathrm{b}}\right), 1.25(9 \mathrm{H}, \mathrm{s}, 3 \times \mathrm{Me}) ; d_{\mathrm{C}}(100 \mathrm{MHz})$ 195.3 (C, C-4), 177.3 (C, O-CO), 142.5 (C, C-CO), 139.9 (C, C-7), 129.9 (C, $\mathrm{C}-\mathrm{CH}), 128.9$ ( $\mathrm{CH}$ arom), 128.6 ( $\mathrm{CH}$ arom), $127.4(\mathrm{CH}$ arom $), 67.9(\mathrm{CH}, \mathrm{C}-1), 38.6\left(\mathrm{C},{ }^{t} \mathrm{Bu}\right), 34.2\left(\mathrm{CH}_{2}\right.$, $\mathrm{C}-3), 28.1\left(\mathrm{CH}_{2}, \mathrm{C}-2\right), 27.1\left(3 \times \mathrm{CH}_{3},{ }^{t} \mathrm{Bu}\right) ; \mathrm{m} / \mathrm{z}\left(\mathrm{CI}+\mathrm{NH}_{3}\right) 299$ $\left(\mathrm{MH}^{+}+\mathrm{NH}_{3}\right), 297\left(\mathrm{MH}^{+}+\mathrm{NH}_{3}\right), 283\left(\mathrm{MH}^{+}\right), 281\left(\mathrm{MH}^{+}\right), 182$ ( $\mathrm{MH}^{+}-$OPiv), $180\left(\mathrm{MH}^{+}-\right.$OPiv); Anal. calcd. for $\mathrm{C}_{15} \mathrm{H}_{17} \mathrm{O}_{3} \mathrm{Cl}$ : C, 64.17; H, 6.10. Found: C, 64.04; H, 6.25\%.

\section{2-(7-Chloro-4-oxo-1,2,3,4-tetrahydro-1-naphthalenyl)ethyl} acetate $(4 c)$

According to the typical procedure, a solution of xanthate $\mathbf{1 b}$ (3.80 g, $13.8 \mathrm{mmol}$ ) and 3-butenyl acetate $2 \mathbf{b}$ (3.15 g, 27.6 mmol) in DCE $(14 \mathrm{~mL})$ was treated with DLP. A refluxing solution of the crude adduct and CSA $(0.32 \mathrm{~g}, 1.4 \mathrm{mmol})$ in DCE $(140 \mathrm{~mL})$ was then subjected to the described reaction conditions to afford tetralone $\mathbf{4 c}$ as a yellow oil $(50 \%)$ (silica gel, petroleum etherethyl acetate, $9: 1) \cdot m_{\max } / \mathrm{cm}^{-1} 1745(\mathrm{O}-\mathrm{C}$ ? $O), 1691\left(\mathrm{C}\right.$ ?) $; d_{\mathrm{H}}$ $(400 \mathrm{MHz}) 7.96(1 \mathrm{H}, \mathrm{d}, J=8.0 \mathrm{~Hz}, \mathrm{CH}$ arom $), 7.26-7.30(2 \mathrm{H}$, $\mathrm{m}, 2 \times \mathrm{CH}$ arom), 4.15-4.23 (4H, m, $\mathrm{CH}_{2}-\mathrm{OAc}$ and $\left.\mathrm{CH}-\mathrm{CH}_{2}\right)$, $3.06(1 \mathrm{H}, \mathrm{m}, 1-\mathrm{H}), 2.74\left(1 \mathrm{H}, \mathrm{ddd}, J=17.9,11.6,5.1 \mathrm{~Hz}, 3-\mathrm{H}_{\mathrm{a}}\right)$, $2.60\left(1 \mathrm{H}, \mathrm{ddd}, J=18.0,5.2,5.2 \mathrm{~Hz}, 3-\mathrm{H}_{\mathrm{b}}\right), 2.28\left(1 \mathrm{H}, \mathrm{m}, 2-\mathrm{H}_{\mathrm{a}}\right)$, $2.01\left(1 \mathrm{H}, \mathrm{m}, 2-\mathrm{H}_{\mathrm{b}}\right), 2.07\left(3 \mathrm{H}, \mathrm{s}, \mathrm{CO}-\mathrm{CH}_{3}\right) ; d_{\mathrm{C}}(100 \mathrm{MHz}) 196.6$ $(\mathrm{C}, \mathrm{C}$ 目 O) $171.0(\mathrm{C}, \mathrm{O}-\mathrm{CO}), 148.7$ (C, C-7), 139.9 (C, C-CO), $130.2(\mathrm{CH}, \mathrm{C}-\mathrm{CH}), 129.3$ ( $\mathrm{CH}$ arom), $128.1(\mathrm{CH}$ arom), 127.5 ( $\mathrm{CH}$ arom), $62.1\left(\mathrm{CH}_{2}, \mathrm{CH}_{2}-\mathrm{OAc}\right), 42.8(\mathrm{CH}, \mathrm{C}-1), 34.9\left(\mathrm{CH}_{3}\right.$, $\left.\mathrm{OC}(\mathrm{O}) \mathrm{CH}_{3}\right), 34.6\left(\mathrm{CH}_{2}, \mathrm{C}-3\right), 33.1\left(\mathrm{CH}_{2}, \mathrm{CH}-\mathrm{CH}_{2}\right), 26.7(\mathrm{C}-$ 2); $m / z\left(\mathrm{CI}+\mathrm{NH}_{3}\right) 286\left(\mathrm{MH}^{+}+\mathrm{NH}_{3}\right), 284\left(\mathrm{MH}^{+}+\mathrm{NH}_{3}\right), 269$ $\left(\mathrm{MH}^{+}\right), 267\left(\mathrm{MH}^{+}\right)$; Anal. calcd. for $\mathrm{C}_{14} \mathrm{H}_{15} \mathrm{ClO}_{3}: \mathrm{C}, 63.04 ; \mathrm{H}$, 5.67. Found: C, $62.83 ; \mathrm{H}, 5.73 \%$.

\section{5,8-Dimethoxy-4-oxo-1,2,3,4-tetrahydro-1-naphthalenyl pivalate (4d)}

Obtained from xanthate 1c as a yellow solid (52\%) (silica gel, petroleum ether-ethyl acetate, $4: 1)$. mp $102-103{ }^{\circ} \mathrm{C}\left(\mathrm{CH}_{2} \mathrm{Cl}_{2}-\right.$ petroleum ether); $m_{\max } / \mathrm{cm}^{-1} 1728(\mathrm{O}-\mathrm{C}$ 回 $O), 1695\left(\mathrm{C}\right.$ 回); $d_{\mathrm{H}}$ $(400 \mathrm{MHz}) 7.06(1 \mathrm{H}, \mathrm{d}, J=8.8 \mathrm{~Hz}, \mathrm{CH}$ arom $), 6.97(1 \mathrm{H}, \mathrm{d}$, $J=9.2 \mathrm{~Hz}, \mathrm{CH}$ arom $), 6.31(1 \mathrm{H}$, br s, $1-\mathrm{H}), 3.86\left(3 \mathrm{H}, \mathrm{s}, \mathrm{OCH}_{3}\right)$, $3.77\left(3 \mathrm{H}, \mathrm{s}, \mathrm{OCH}_{3}\right), 2.82\left(1 \mathrm{H}, \mathrm{ddd}, J=15.2,5.1,5.1 \mathrm{~Hz}, 3-\mathrm{H}_{\mathrm{a}}\right)$, $2.53\left(1 \mathrm{H}\right.$, ddd, $\left.J=16.4,16.4,4.8 \mathrm{~Hz}, 3-\mathrm{H}_{\mathrm{b}}\right), 2.33(1 \mathrm{H}$, dddd, $\left.J=14.4,14.4,2.3,0.0 \mathrm{~Hz}, 2-\mathrm{H}_{\mathrm{a}}\right), 2.17$ (1H, dddd, $J=14.2,14.2$, 3.6, $\left.3.6 \mathrm{~Hz}, 2-\mathrm{H}_{\mathrm{b}}\right), 1.13\left(9 \mathrm{H}, \mathrm{s},{ }^{t} \mathrm{Bu}\right) ; d_{\mathrm{C}}(100 \mathrm{MHz}) 197.2(\mathrm{C}$, $C$ 回 $O), 177.6(\mathrm{C}, \mathrm{O}-\mathrm{CO}), 153.4(\mathrm{C}, C-\mathrm{OMe}), 150.6(\mathrm{C}, C-\mathrm{OMe})$, 130.0 (C, $C-\mathrm{CO}), 122.6(\mathrm{C}, \mathrm{C}-\mathrm{CH}), 116.8(\mathrm{CH}$ arom $), 113.5(\mathrm{CH}$ arom), $63.7(\mathrm{C}-1), 56.5\left(\mathrm{CH}_{3}, \mathrm{OMe}\right), 56.2\left(\mathrm{CH}_{3}, \mathrm{OMe}\right), 38.9(\mathrm{C}$, $\left.{ }^{t} \mathrm{Bu}\right), 34.9\left(\mathrm{CH}_{2}, \mathrm{C}-3\right), 27.1\left(3 \times \mathrm{CH}_{3},{ }^{t} \mathrm{Bu}\right), 26.9\left(\mathrm{CH}_{2}, \mathrm{C}-2\right) ; \mathrm{m} / \mathrm{z}$ $\left(\mathrm{CI}+\mathrm{NH}_{3}\right) 307\left(\mathrm{MH}^{+}\right), 205\left(\mathrm{MH}^{+}-\mathrm{OPiv}\right)$; Anal. calcd. for $\mathrm{C}_{17} \mathrm{H}_{22} \mathrm{O}_{5}$ : C, 66.65; H, 7.24. Found: C, 66.32; H, 7.25\%.

\section{Diethyl 5-[(2,2-dimethylpropanoyl)oxy]-2,8-dioxo-5,6,7,8- tetrahydro- $1 H$-naphtho[2,3- $d]$ imidazole-1,3(2H)-dicarboxylate (4e)}

Obtained from xanthate $\mathbf{1 d}^{14}$ as a white solid (54\%) (silica gel, petroleum ether-ethyl acetate, $8: 2), \mathrm{mp} 120-123{ }^{\circ} \mathrm{C}$ (AcOEt); $m_{\max } / \mathrm{cm}^{-1} 1791(\mathrm{O}-C$ 回 $O), 1752(\mathrm{O}-C$ Q $O), 1731(\mathrm{O}-C$ @ $O)$, $1694(\mathrm{C}$ O O $) ; d_{\mathrm{H}}(400 \mathrm{MHz}) 8.10(2 \mathrm{H}, \mathrm{s}, 2 \times \mathrm{CH}$ arom $), 6.36$ (1H, dd,

$J=7.2,4.0 \mathrm{~Hz}, 5-\mathrm{H}), 4.46-4.55\left(4 \mathrm{H}, \mathrm{m}, 2 \times \mathrm{COOCH}_{2} \mathrm{CH}_{3}\right), 2.81$ $\left(1 \mathrm{H}, \mathrm{m}, 7-\mathrm{H}_{\mathrm{a}}\right), 2.70\left(1 \mathrm{H}, \mathrm{m}, 7-\mathrm{H}_{\mathrm{b}}\right), 2.50\left(1 \mathrm{H}, \mathrm{m}, 6-\mathrm{H}_{\mathrm{a}}\right), 2.14(1 \mathrm{H}$, $\left.\mathrm{m}, 6-\mathrm{H}_{\mathrm{b}}\right), 1.47\left(6 \mathrm{H}, \mathrm{dd}, J=15.2,6.8 \mathrm{~Hz}, 2 \times \mathrm{COOCH}_{2} \mathrm{CH}_{3}\right)$, $1.14\left(9 \mathrm{H}, \mathrm{s},{ }^{t} \mathrm{Bu}\right) ; d_{\mathrm{C}}(100 \mathrm{MHz}) 195.4(\mathrm{C}, \mathrm{C}$ 回 $), 177.3(\mathrm{C}), 149.6$ $(2 \times \mathrm{C}), 147.7(\mathrm{C}), 131.4(\mathrm{C}, C-\mathrm{N}), 129.5(\mathrm{C}, C-\mathrm{N}), 126.6(\mathrm{C}$, $C-\mathrm{CO}), 125.1(\mathrm{CH}$ arom $), 123.6(\mathrm{C}, \mathrm{C}-\mathrm{CH}), 114.5(\mathrm{CH}$ arom $)$, $66.8(\mathrm{CH}, \mathrm{C}-5), 65.6\left(\mathrm{CH}_{3}\right), 64.6\left(\mathrm{CH}_{3}\right), 39.0\left(\mathrm{C},{ }^{t} \mathrm{Bu}\right), 33.6$ $\left(\mathrm{CH}_{2}, \mathrm{C}-7\right), 27.4\left(\mathrm{CH}_{2}, \mathrm{C}-6\right), 26.9\left(3 \times \mathrm{CH}_{3},{ }^{t} \mathrm{Bu}\right), 14.1\left(2 \times \mathrm{CH}_{3}\right.$, $\left.2 \times \mathrm{CO}_{2} \mathrm{Et}\right) ; \mathrm{m} / \mathrm{z}\left(\mathrm{CI}+\mathrm{NH}_{3}\right) 464\left(\mathrm{MH}^{+}+\mathrm{NH}_{3}\right), 447\left(\mathrm{MH}^{+}\right)$.

\section{1-(2,2-Dimethylpropanoyl)-8-oxo-5,6,7,8-tetrahydro- $1 \mathrm{H}$ - benzo[f $]$ indol-5-yl pivalate (4f)}

Obtained from xanthate $1 \mathbf{1}^{13}$ as a white solid (41\%) (silica gel, petroleum ether-ethyl acetate, $95: 5), \mathrm{mp} 149-151^{\circ} \mathrm{C}$ (petroleum ether); $m_{\max } / \mathrm{cm}^{-1} 1724(\mathrm{O}-C$ 回 $O), 1682(\mathrm{C}$ 回 $) ; d_{\mathrm{H}}(400 \mathrm{MH}$ $8.06(1 \mathrm{H}, \mathrm{d}, J=8.0 \mathrm{~Hz}, \mathrm{CH}$ arom $), 7.75(1 \mathrm{H}, \mathrm{d}, J=3.6 \mathrm{~Hz}$, $\mathrm{C} H$ arom $), 7.63(1 \mathrm{H}, \mathrm{d}, J=8.4 \mathrm{~Hz}, \mathrm{CH}$ arom $), 6.63(1 \mathrm{H}, \mathrm{d}$, $J=3.6 \mathrm{~Hz}, \mathrm{CH}$ arom), 6.44 (br s, $5-\mathrm{H}), 2.98(1 \mathrm{H}, \mathrm{ddd}, J=18.4$, 12.6, 6.0 Hz, 7-Ha $), 2.70\left(1 \mathrm{H}, \mathrm{m}, 7-\mathrm{H}_{\mathrm{b}}\right), 2.35-2.49\left(2 \mathrm{H}, \mathrm{m}, 6-\mathrm{H}_{2}\right)$, $1.58\left(9 \mathrm{H}, \mathrm{s},{ }^{t} \mathrm{Bu}\right), 1.06\left(9 \mathrm{H}, \mathrm{s},{ }^{t} \mathrm{Bu}\right) ; d_{\mathrm{C}}(100 \mathrm{MHz}) 197.9(\mathrm{C}$, $C$ 回 $O), 180.1(\mathrm{C}, \mathrm{N}-C \mathrm{O}), 177.5(\mathrm{C}, \mathrm{O}-C \mathrm{O}), 136.0(\mathrm{C}, C-\mathrm{N}-\mathrm{Piv})$, 133.6 (C, $C-\mathrm{CO}), 130.2(\mathrm{C}, C-\mathrm{C}-\mathrm{N}), 130.0(C \mathrm{H}$ arom $), 129.3$ 
(C, C-CH), 123.1 ( $\mathrm{CH}$ arom), 121.7 ( $\mathrm{CH}$ arom), 107.1 (CH arom), $65.8(\mathrm{CH}, \mathrm{C}-5), 42.1\left(\mathrm{C}, \mathrm{N}-\mathrm{CO}-\mathrm{C}\left(\mathrm{CH}_{3}\right)_{3}\right), 38.8(\mathrm{C}$, $\left.\mathrm{O}-\mathrm{CO}-\mathrm{C}\left(\mathrm{CH}_{3}\right)_{3}\right), 32.6\left(\mathrm{CH}_{2}, \mathrm{C}-7\right), 29.0\left(3 \times \mathrm{CH}_{3}, \mathrm{~N}-\mathrm{CO}-\right.$ $\left.\mathrm{C}\left(\mathrm{CH}_{3}\right)_{3}\right), 27.5\left(\mathrm{CH}_{2}, \mathrm{C}-6\right), 27.1\left(3 \times \mathrm{CH}_{3}, \mathrm{O}-\mathrm{CO}-\mathrm{C}\left(\mathrm{CH}_{3}\right)_{3}\right) ; \mathrm{m} / z$ $\left(\mathrm{CI}+\mathrm{NH}_{3}\right) 387\left(\mathrm{MH}^{+}+\mathrm{NH}_{3}\right), 370\left(\mathrm{MH}^{+}\right), 268\left(\mathrm{MH}^{+}-\right.$OPiv $)$.

\section{Aromatisation of substituted $a$-tetralones}

Method A. 6-Fluoro-1-naphthol (5). A solution of tetralone 4a $(0.1 \mathrm{~g}, 0.378 \mathrm{mmol})$ and of PTSA $\cdot \mathrm{H}_{2} \mathrm{O}(0.21 \mathrm{~g}, 1.13 \mathrm{mmol})$ in $12.5 \mathrm{~mL}$ of toluene was refluxed for $3 \mathrm{~h}$ with a Dean-Stark apparatus. When the starting material was totally consumed, the reaction mixture was allowed to cool to room temperature, neutralised with saturated aqueous $\mathrm{Na}_{2} \mathrm{CO}_{3}$, extracted with $\mathrm{CH}_{2} \mathrm{Cl}_{2}$, dried $\left(\mathrm{Na}_{2} \mathrm{SO}_{4}\right)$ and evaporated under reduced pressure. The residue was purified by flash column chromatography (silica gel, petroleum ether-ethyl acetate, $9: 1)$ to give naphthol $\mathbf{5}(84 \%)$ as a pale brown solid. $\mathrm{mp} 112-113{ }^{\circ} \mathrm{C}$ (petroleum ether); $m_{\max } / \mathrm{cm}^{-1}$ $3605(\mathrm{OH}) ; d_{\mathrm{H}}(400 \mathrm{MHz}) 8.21(1 \mathrm{H}, \mathrm{dd}, J=10.0,6.0 \mathrm{~Hz}, \mathrm{CH}$ arom), $7.42(1 \mathrm{H}, \mathrm{dd}, J=14.0,6.0 \mathrm{~Hz}, \mathrm{CH}$ arom $), 7.22-7.38$ $(3 \mathrm{H}, \mathrm{m}, 3 \times \mathrm{CH}$ arom), $6.75(1 \mathrm{H}, \mathrm{d}, J=4.0 \mathrm{~Hz}, \mathrm{CH}$ arom $) ; d$ $(100 \mathrm{MHz}) 161.3\left(\mathrm{C}, \mathrm{d},{ }^{1} J_{\mathrm{CF}}=244.6 \mathrm{~Hz}, C-\mathrm{F}\right), 151.8(\mathrm{C}, \mathrm{C}-1)$, $135.9\left(\mathrm{C}, \mathrm{d},{ }^{3} \mathrm{~J}=\mathrm{FF} 9.3 \mathrm{~Hz}, C-\mathrm{CH}\right), 127.3(\mathrm{CH}$ arom $), 124.7$ $\left(\mathrm{CH}, \mathrm{d},{ }^{3} J_{\mathrm{CF}}=9.2 \mathrm{~Hz}, \mathrm{CH}\right.$ arom $), 121.6(\mathrm{C}, \mathrm{C}-\mathrm{C}-\mathrm{OH}), 120.1$ $\left(\mathrm{CH}, \mathrm{d},{ }^{4} J_{\mathrm{C}} \overline{\mathrm{F}} 5.1 \mathrm{~Hz}, \mathrm{CH}\right.$ arom $), 115.5\left(\mathrm{CH}, \mathrm{d},{ }^{2} J \overline{\mathrm{CF}} 25.1 \mathrm{~Hz}\right.$, $\mathrm{CH}$ arom $), 110.7\left(\mathrm{CH}, \mathrm{d},{ }^{2} J_{\mathrm{CF}}=20.3 \mathrm{~Hz}, \mathrm{CH}\right.$ arom $), 107.9$ $\left(\mathrm{CH}, \mathrm{d},{ }^{5} J_{\mathrm{CF}}=2.2 \mathrm{~Hz}, \mathrm{CH}\right.$ arom $) ; m / z\left(\mathrm{CI}+\mathrm{NH}_{3}\right) 163\left(\mathrm{MH}^{+}\right)$; $\mathrm{m} / \mathrm{z}$ (rel intensity) $162\left(\mathrm{M}^{+}, 100\right)$; HRMS calcd for $\mathrm{C}_{15} \mathrm{H}_{13} \mathrm{ClO}_{2}$ : 162.048093; found 162.047009.

\section{Diethyl 5-hydroxy-2-oxo-1H-naphtho[2,3-d]imidazole-} 1,3(2H)-dicarboxylate (6). A solution of tetralone $4 \mathrm{e}(40 \mathrm{mg}$, $0.09 \mathrm{mmol})$ and PTSA $\cdot \mathrm{H}_{2} \mathrm{O}(44 \mathrm{mg}, 0.23 \mathrm{mmol})$ in toluene $(3 \mathrm{ml})$ was stirred at reflux for $2.5 \mathrm{~h}$ using a Dean-Stark apparatus. The reaction mixture was allowed to cool to room temperature, diluted with a saturated sodium bicarbonate solution, and extracted with dichloromethane. The combined organic layers were dried over $\mathrm{MgSO}_{4}$, filtered and concentrated. The residue was purified by column chromatography (silica gel, petroleum ether-ethyl acetate, $75: 25$ ) to afford compound 6 (23 mg, 74\%) as an amorphous solid; $m_{\max } / \mathrm{cm}^{-1} 3608(\mathrm{OH})$, $1797(C$ 回O $), 1748(C$ 回 $), 1714(C$ 回 $) ; d_{\mathrm{H}}(400 \mathrm{MHz}) 8.22$ $(1 \mathrm{H}, \mathrm{d}, J=9.2 \mathrm{~Hz}), 8.14(1 \mathrm{H}, \mathrm{d}, J=9.2 \mathrm{~Hz}), 7.35-7.37 \quad(2 \mathrm{H}$, $\mathrm{m}), 6.84(1 \mathrm{H}, \mathrm{m}), 6.84(1 \mathrm{H}, \mathrm{m}), 5.90(1 \mathrm{H}, \mathrm{br} \mathrm{s}, \mathrm{OH}), 4.55-4.63$ $\left(4 \mathrm{H}, \mathrm{m}, 2 \times \mathrm{CH}_{2} \mathrm{CH}_{3}\right), 1.51-1.52\left(6 \mathrm{H}, \mathrm{m}, 2 \times \mathrm{CH}_{2} \mathrm{CH}_{3}\right) ; d$ $(100 \mathrm{MHz}) 203.9\left(\mathrm{C}, \mathrm{N}-\mathrm{CO}_{2} \mathrm{Et}\right), 200.3\left(\mathrm{C}, \mathrm{N}-\mathrm{CO}_{2} \mathrm{Et}\right), 190.6$ $(\mathrm{C}, \mathrm{N}-\mathrm{CO}-\mathrm{N}), 152.1(\mathrm{C}), 150.0(\mathrm{C}), 148.6$ (C), $126.8(\mathrm{CH}$ arom), $122.4(\mathrm{C}), 121.5(\mathrm{C}), 120.0(\mathrm{CH}$ arom $), 114.5(\mathrm{CH}$ arom), 112.6 ( $\mathrm{CH}$ arom $), 107.9(\mathrm{CH}$ arom $), 65.2\left(\mathrm{CH}_{2}\right.$, $\left.\mathrm{CH}_{2} \mathrm{CH}_{3}\right), 64.2\left(\mathrm{CH}_{2}, \mathrm{CH}_{2} \mathrm{CH}_{3}\right), 14.1\left(2 \times \mathrm{CH}_{3}, 2 \times\right.$ $\left.\mathrm{CH}_{2} \mathrm{CH}_{3}\right) ; \mathrm{m} / \mathrm{z}\left(\mathrm{CI}+\mathrm{NH}_{3}\right) 345\left(\mathrm{MH}^{+}\right), 362\left(\mathrm{M}^{+}+\mathrm{NH}_{3}\right) ; \mathrm{m} / \mathrm{z}$ (rel intensity) $344\left(\mathrm{M}^{+}, 79\right), 272\left(\mathrm{M}^{+}-1-\mathrm{C}_{3} \mathrm{H}_{5} \mathrm{O}_{2}, 30\right), 200$ (100); HRMS calcd for $\mathrm{C}_{17} \mathrm{H}_{16} \mathrm{~N}_{2} \mathrm{O}_{6}$ : 344.100836; found 344.101677.

2-Bromo-6-fluoro-1-naphthol (8). Pyridinium bromide perbromide (52 $\mathrm{mg}, 0.16 \mathrm{mmol}$ ) was added to a solution of tetralone $4 \mathbf{a}(43 \mathrm{mg}, 0.16 \mathrm{mmol})$ in acetic acid $(1.6 \mathrm{ml})$ and the mixture was then stirred for $2 \mathrm{~h}$ at room temperature. It was diluted with a saturated sodium carbonate solution, and extracted with ethyl acetate. The combined organic layers were dried, filtered and concentrated. The residue was dissolved in toluene $(5.6 \mathrm{ml})$ and PTSA $\cdot \mathrm{H}_{2} \mathrm{O}(91 \mathrm{mg}, 0.48 \mathrm{mmol})$ was added. The solution was stirred at reflux for $3.5 \mathrm{~h}$ using a Dean-Stark apparatus. The reaction mixture was allowed to cool to room temperature, diluted with a saturated sodium bicarbonate solution, and extracted with dichloromethane. The combined organic layers were dried, filtered and concentrated. The residue was purified by column chromatography (silica gel, petroleum ether-ethyl acetate, 95 : 5) to afford compound $\mathbf{8}(26 \mathrm{mg}, 69 \%)$ as a white solid. $\mathrm{mp}$ $60-65^{\circ} \mathrm{C}\left(\mathrm{CH}_{2} \mathrm{Cl}_{2}\right) ; m_{\max } / \mathrm{cm}^{-1} 3517(\mathrm{OH}) ; d_{\mathrm{H}}(400 \mathrm{MHz}) 8.24$ $(1 \mathrm{H}, \mathrm{dd}, J=9.2,5.6 \mathrm{~Hz}), 7.49(1 \mathrm{H}, \mathrm{d}, J=8.8 \mathrm{~Hz}), 7.39(1 \mathrm{H}$, $\mathrm{dd}, J=9.6,2.4 \mathrm{~Hz}), 7.29(1 \mathrm{H}, \mathrm{ddd}, J=9.2,9.2,2.8 \mathrm{~Hz}), 7.24$ $(1 \mathrm{H}, \mathrm{d}, J=8.8 \mathrm{~Hz}), 6.01(1 \mathrm{H}, \mathrm{br} \mathrm{s}, \mathrm{OH}) ; d_{\mathrm{C}}(100 \mathrm{MHz}) 162.5$ (C), $160.1(\mathrm{C}), 148.4(\mathrm{C}), 134.8(2 \times \mathrm{C}), 129.7(\mathrm{CH}), 125.2$ $\left(\mathrm{CH},{ }^{3} J_{\mathrm{CF}}=9.2 \mathrm{~Hz}\right), 120.6\left(\mathrm{CH},{ }^{4} J_{\mathrm{CF}}=5.1 \mathrm{~Hz}\right), 116.1(\mathrm{CH}$, $\left.{ }^{2} J_{\mathrm{CF}}=16.4 \mathrm{~Hz}\right), 110.8\left(\mathrm{CH},{ }^{2} J_{\mathrm{CF}}=20.7 \mathrm{~Hz}\right) ; \mathrm{m} / \mathrm{z}\left(\mathrm{CI}+\mathrm{NH}_{3}\right)$ $240\left(\mathrm{M}^{+-}-1\right), 257\left(\mathrm{M}^{+-} 1+\mathrm{NH}_{3}\right) ; \mathrm{m} / z$ (rel intensity) $242\left(\mathrm{M}^{+}\right.$, 11), $240\left(\mathrm{M}^{+}, 100\right)$; HRMS calcd for $\mathrm{C}_{10} \mathrm{H}_{6} \mathrm{FBrO}$ : 241.956558; found 241.953297. Anal. Calcd. for $\mathrm{C}_{10} \mathrm{H}_{6} \mathrm{FBrO}$ : C, 49.83; $\mathrm{H}$, 2.51. Found: C, $50.26 ; \mathrm{H}, 3.02 \%$.

2,2-Dimethyl-propionic acid 3-ethoxythiocarbonylsulfanyl-7fluoro-4-oxo-1,2,3,4-tetrahydro-naphthalen-1-yl ester (15). To a solution of tetralone $4 \mathbf{a}(0.2 \mathrm{~g}, 0.75 \mathrm{mmol})$ in acetic acid $(7.5 \mathrm{~mL})$ at room temperature was slowly added pyridinium bromide perbromide $(0.29 \mathrm{~g}, 0.91 \mathrm{mmol})$. The reaction was stirred at room temperature for $4 \mathrm{~h}$, neutralised with saturated aqueous $\mathrm{Na}_{2} \mathrm{CO}_{3}$, extracted with dichloromethane, dried $\left(\mathrm{Na}_{2} \mathrm{SO}_{4}\right)$ and evaporated. To a cold solution $\left(0^{\circ} \mathrm{C}\right)$ of the residue in acetone $(1.5 \mathrm{~mL})$ was then slowly added potassium $O$-ethyl xanthate $(0.13 \mathrm{~g}, 0.83 \mathrm{mmol})$. The reaction mixture was stirred at $0{ }^{\circ} \mathrm{C}$ for a further $1 \mathrm{~h}$, the solvent was evaporated and the resulting mixture partitioned between water and $\mathrm{CH}_{2} \mathrm{Cl}$. The aqueous phase was extracted with $\mathrm{CH}_{2} \mathrm{Cl}_{2}$, the combined organic extracts were dried over $\mathrm{Na}_{2} \mathrm{SO}_{4}$ and concentrated under reduced pressure. Flash chromatography of the residue (silica gel, petroleum ether-ethyl acetate, $99: 1$ to $95: 5)$, gave xanthate $\mathbf{1 5}(68 \%$ overall yield) as a yellow oil and an inseparable mixture of diastereoisomers (ratio 2:1). $m_{\text {max }} / \mathrm{cm}^{-1} 1734(\mathrm{O}-C$ 回O), 1695 (C回O), 1235 (S-C? $1052(S-C$ 回 $) ; d_{\mathrm{H}}(400 \mathrm{MHz}) 8.10-8.16(1 \mathrm{H}, \mathrm{m}, \mathrm{CH}$ arom), 7.12-7.20 (2H, m, $2 \times \mathrm{CH}$ arom $), 6.23(1 \mathrm{H}, \mathrm{dd}, J=10.4$, $4.4 \mathrm{~Hz}, \mathrm{CH} b-\mathrm{OPiv}), 6.08$ (1H, dd, $J=2.8,2.8 \mathrm{~Hz}, \mathrm{CHa}-\mathrm{OPiv})$, $5.16(1 \mathrm{H}, \mathrm{dd}, J=12.4,4.8 \mathrm{~Hz}, \mathrm{CHa}-\mathrm{S}), 4.87(1 \mathrm{H}, \mathrm{dd}, J=13.2$, $4.8 \mathrm{~Hz}, \mathrm{CHb}-\mathrm{S}), 4.61-4.67$ (2H, m, O-CHa and $\mathrm{O}-\mathrm{CH} b), 2.94$ $\left(1 \mathrm{H}, \mathrm{ddd}, J=12.0,4.8,4.8 \mathrm{~Hz}, \mathrm{CH}_{2} b\right), 2.84(1 \mathrm{H}$, ddd, $J=14.0$, $\left.4.0,4.0 \mathrm{~Hz}, \mathrm{CH}_{2} a\right), 2.64\left(1 \mathrm{H}, \mathrm{ddd}, J=13.2,13.2,2.8 \mathrm{~Hz}, \mathrm{CH} \square_{2} a\right)$, $2.41\left(1 \mathrm{H}, \mathrm{q}, J=11.5 \mathrm{~Hz}, \mathrm{CH} \square_{2} b\right), 1.38(3 \mathrm{H}, \mathrm{dd}, J=7.2,7.2 \mathrm{~Hz}$, $\mathrm{CH}_{2}-\mathrm{CH}_{3} a$ and $\left.\mathrm{CH}_{2}-\mathrm{CH}_{3} b\right), 1.30\left(3 \mathrm{H}, \mathrm{s},\left(\mathrm{CH}_{3} b\right)_{3}\right), 1.22(6 \mathrm{H}$, s, $\left.\left(\mathrm{CH}_{3} a\right)_{3}\right) ; d_{\mathrm{C}}(100 \mathrm{MHz}) 212.5(\mathrm{Ca}$ 回 $), 212.0(\mathrm{Cb}$ 回 $), 1966$ $(\mathrm{Ca}$ 回O), $189.9(\mathrm{Cb}$ 回 $), 177.7(\mathrm{O}-\mathrm{CaO}$ and $\mathrm{O}-\mathrm{CbO}), 166.4$ $\left(\mathrm{C}, \mathrm{d}, C b-\mathrm{F},{ }^{1} J_{\mathrm{CF}}=256 \mathrm{~Hz}\right), 166.1\left(\mathrm{C}, \mathrm{d}, C a-\mathrm{F},{ }^{1} J_{\mathrm{CF}}=255 \mathrm{~Hz}\right)$, $142.4(\mathrm{Cb}-\mathrm{CO}), 142.3(\mathrm{Ca}-\mathrm{CO}), 131.4(3 \times \mathrm{CH}$ arom $), 128.4$ $(\mathrm{Ca}-\mathrm{CH}$ and $\mathrm{Cb}-\mathrm{CH}), 117.4\left(\mathrm{CH}, \mathrm{d},{ }^{2} J_{\mathrm{CF}}=21.8 \mathrm{~Hz}, \mathrm{CH}\right.$ arom $), 116.4\left(\mathrm{CH}, \mathrm{d},{ }^{2} J_{\mathrm{CF}}=22.1 \mathrm{~Hz}, \mathrm{CH}\right.$ arom $), 112.9(\mathrm{CH}, \mathrm{d}$, ${ }^{2} J_{\mathrm{CF}}=22.9 \mathrm{~Hz}, C \mathrm{H}$ arom $), 70.8\left(\mathrm{O}-\mathrm{CH}_{2} a\right.$ and $\left.\mathrm{O}-\mathrm{CH}_{2} b\right), 67.9$ ( $\mathrm{CH} a$-OPiv), 67.8 ( $\mathrm{CH} b-\mathrm{OPiv}), 54.0(\mathrm{CH} a-\mathrm{S}), 52.6(\mathrm{CH} b-\mathrm{S})$, $38.8\left(\mathrm{Ca}\left(\mathrm{CH}_{3}\right)_{3}\right.$

and $\left.\mathrm{Cb}\left(\mathrm{CH}_{3}\right)_{3}\right), 36.0\left(\mathrm{CH}_{2} b\right), 35.2\left(\mathrm{CH}_{2} a\right), 27.2\left(\left(\mathrm{CH}_{3} a\right)_{3}\right.$ and $\left.\left(\mathrm{CH}_{3} b\right)_{3}\right), 13.8\left(\mathrm{CH}_{2}-\mathrm{CH}_{2} a\right.$ and $\left.\mathrm{CH}_{2}-\mathrm{CH}_{3} b\right) ; \mathrm{m} / \mathrm{z}\left(\mathrm{CI}+\mathrm{NH}_{3}\right)$ $385\left(\mathrm{MH}^{+}\right), 283\left(\mathrm{MH}^{+}-\mathrm{OPiv}\right)$.

7-Fluoronaphtho[2,1-d][1,3]oxathiole-2-thione (16). According to the general method A, this compound was prepared from tetralone derivative $\mathbf{1 5}$ to give compound $\mathbf{1 6}(89 \%)$ as an orange solid. mp $157-159^{\circ} \mathrm{C}\left(\mathrm{CH}_{2} \mathrm{Cl}_{2}\right.$-petroleum ether $) ; m_{\max } / \mathrm{cm}^{-1} 1189$ (C回S ); $d_{\mathrm{H}}(400 \mathrm{MHz}) 8.25(1 \mathrm{H}, \mathrm{dd}, J=8.8,5.6 \mathrm{~Hz}, \mathrm{CH}$ arom), $7.76(1 \mathrm{H}, \mathrm{d}, J=8.4 \mathrm{~Hz}, \mathrm{CH}$ arom $), 7.54(1 \mathrm{H}, \mathrm{d}, J=9.6 \mathrm{~Hz}$, $\mathrm{C} H$ arom), $7.47(1 \mathrm{H}, \mathrm{d}, J=8.4 \mathrm{~Hz}, \mathrm{C} H$ arom $), 7.43(1 \mathrm{H}, \mathrm{d}, J$ $=8.0 \mathrm{~Hz}, \mathrm{CH}$ arom $) ; d_{\mathrm{C}}(100 \mathrm{MHz}) 201.5(\mathrm{C}, \mathrm{C}$ 回 $), 1613(\mathrm{C}$, $\left.\mathrm{d},{ }^{1} J_{\mathrm{CF}}=247.0 \mathrm{~Hz}, C-\mathrm{F}\right), 150.5(\mathrm{C}, C-\mathrm{O}), 133.9(\mathrm{C}, \mathrm{d}$, $\left.{ }^{3} J_{\mathrm{CF}}=9.6 \mathrm{~Hz}, \mathrm{CH}-\mathrm{C}-\mathrm{CH}\right), 125.8(\mathrm{CH}$ arom $), 123.9(\mathrm{CH}, \mathrm{d}$, ${ }^{3} J_{\mathrm{CF}}=9.0 \mathrm{~Hz}, C \mathrm{H}$ arom $), 120.4(\mathrm{C}, C-\mathrm{C}-\mathrm{O}), 118.8(C \mathrm{H}$ arom $)$, $118.5\left(\mathrm{CH}, \mathrm{d},{ }^{2} J_{\mathrm{CF}}=25.9 \mathrm{~Hz}, \mathrm{CH}\right.$ arom $), 117.3(\mathrm{C}, C-\mathrm{S}), 111.9$ $\left(\mathrm{CH}, \mathrm{d},{ }^{2} J_{\mathrm{CF}}=26.0 \mathrm{~Hz}, \mathrm{CH}\right.$ arom $) ; \mathrm{m} / z\left(\mathrm{CI}+\mathrm{NH}_{3}\right) 237\left(\mathrm{MH}^{+}\right)$; $\mathrm{m} / \mathrm{z}$ (rel intensity) $236\left(\mathrm{M}^{+}, 12\right), 176\left(\mathrm{M}^{+}-\mathrm{COS}, 100\right)$; HRMS calcd for $\mathrm{C}_{11} \mathrm{H}_{5} \mathrm{FOS}_{2}$ : 235.973215; found 235.973385.

7-Fluoro-4-(2-methoxy-2-oxoethylidene)-1,2,3,4-tetrahydro1-naphthalenyl pivalate (22). Methyl (diethoxyphosphoryl)acetate $(0.36 \mathrm{~mL}, 1.96 \mathrm{mmol})$ was added dropwise to a cooled suspension of $\mathrm{NaH}$ (80\% dispersion in mineral oil, $60 \mathrm{mg}$, $1.98 \mathrm{mmol})$ in anhydrous toluene $(1.2 \mathrm{~mL})$. The mixture was then stirred for $1 \mathrm{~h}$ at $60^{\circ} \mathrm{C}$ to ensure the formation of the ylide. 
The formed solution was cooled, and a solution of tetralone $4 \mathbf{a}$ $(368 \mathrm{mg}, 1.39 \mathrm{mmol})$ in anhydrous toluene $(1.2 \mathrm{ml})$ was added dropwise. The mixture was refluxed for $3 \mathrm{~h}$, cooled to room temperature, and then washed with cold water and extracted with dichloromethane. The separated organic layer was dried over $\mathrm{MgSO}_{4}$, and the solvent was evaporated under reduced pressure. The crude residue was chromatographed (petroleum ether-ethyl acetate, $95: 5)$ to yield $\mathbf{2 2}(272 \mathrm{mg}, 61 \%)$ as a colourless oil. $m_{\max } / \mathrm{cm}^{-1} 1728$ (C回), 1605 (C回O); $d_{\mathrm{H}}(400 \mathrm{MHz})$ compl mixture of isomers; $d_{\mathrm{C}}(100 \mathrm{MHz})$ complex mixture of isomers; $m / z\left(\mathrm{CI}+\mathrm{NH}_{3}\right) 291\left(\mathrm{MH}^{+}-2 \mathrm{CH}_{3}\right), 308\left(\mathrm{MH}^{+}+\mathrm{NH}_{3}-2 \mathrm{CH}_{3}\right)$, $323\left(\mathrm{MH}^{+}+\mathrm{NH}_{3}-\mathrm{CH}_{3}\right), 338\left(\mathrm{MH}^{+}+\mathrm{NH}_{3}\right)$.

Methyl (6-fluoro-1-naphthyl)acetate (23). According to the general method A, this compound was prepared from derivative $\mathbf{2 2}$ to give compound $\mathbf{2 3}(67 \%)$ as a solid amorphous. $m_{\max } / \mathrm{cm}^{-1}$ $1738(\mathrm{C}$ 目 $), 1634(\mathrm{C}$ 回 $) ; d_{\mathrm{H}}(400 \mathrm{MHz}) 8.01(1 \mathrm{H}, \mathrm{dd}, J=52$ $9.2 \mathrm{~Hz}, \mathrm{CH}$ arom), $7.74(1 \mathrm{H}, \mathrm{d}, J=8.4 \mathrm{~Hz}, \mathrm{CH}$ arom), 7.44-7.50 (2H, m, $2 \times \mathrm{CH}$ arom), $7.38(1 \mathrm{H}, \mathrm{d}, J=6.8 \mathrm{~Hz}, \mathrm{CH}$ arom), 7.32 $(1 \mathrm{H}, \mathrm{m}, \mathrm{CH}$ arom $), 4.17\left(1 \mathrm{H}, \mathrm{d}, J=14.4 \mathrm{~Hz}, \mathrm{CH}_{2}\right), 4.15(1 \mathrm{H}$, $\left.\mathrm{d}, J=14.4 \mathrm{~Hz}, \mathrm{CH}_{2}\right), 4.06\left(3 \mathrm{H}, \mathrm{s}, \mathrm{CH}_{3}\right) ; d_{\mathrm{C}}(100 \mathrm{MHz}) 161.6$ $(C), 134.8(C), 130.9(C), 129.1(C), 127.8(C), 126.6(2 \times C H$ arom $), 118.7(2 \times \mathrm{CH}$ arom $), 116.6(2 \times \mathrm{CH}$ arom $), 61.1\left(\mathrm{CH}_{3}\right)$, $39.4\left(\mathrm{CH}_{2}\right) ; \mathrm{m} / \mathrm{z}\left(\mathrm{CI}+\mathrm{NH}_{3}\right) 218\left(\mathrm{M}^{+}\right), 233\left(\mathrm{M}^{+}+\mathrm{NH}\right) . \mathrm{m} / \mathrm{z}$ $\left(\mathrm{CI}+\mathrm{NH}_{3}\right) 218\left(\mathrm{M}^{+}\right), 233\left(\mathrm{M}^{+}+\mathrm{NH}\right) ; \mathrm{m} / z$ (rel intensity) 218 $\left(\mathrm{M}^{+}, 15\right), 159\left(\mathrm{M}^{+}-\mathrm{C}_{2} \mathrm{H}_{3} \mathrm{O}_{2}, 100\right)$; HRMS calcd for $\mathrm{C}_{13} \mathrm{H}_{11} \mathrm{FO}_{2}$ : 218.074308; found 218.073933.

Method B. 2-Bromo-5,8-dimethoxy-1-naphthol (10). To a stirred solution of tetralone $9(0.2 \mathrm{~g}, 0.65 \mathrm{mmol})$ in acetic acid $(6.5 \mathrm{~mL})$ at room temperature was added slowly pyridinium bromide perbromide $(0.21 \mathrm{~g}, 0.65 \mathrm{mmol})$. The mixture was stirred at room temperature for $1 \mathrm{~h}$, neutralised with saturated aqueous $\mathrm{Na}_{2} \mathrm{CO}_{3}$, extracted with $\mathrm{CH}_{2} \mathrm{Cl}_{2}$, dried over anhydrous $\mathrm{Na}_{2} \mathrm{SO}_{4}$ and concentrated under reduced pressure. The residue was purified by flash column chromatography (silica gel, petroleum ether-ethyl acetate, $8: 2$ ) to give directly the naphthol $10(75 \%)$ as a brown-green solid. mp $137-138{ }^{\circ} \mathrm{C}\left(\mathrm{CH}_{2} \mathrm{Cl}_{2}-\right.$ petroleum ether) (lit., ${ }^{15} 134^{\circ} \mathrm{C}$ ).

Method C. 7-Fluoro-4-hydroxy-1-naphthyl pivalate (11). A mixture of residue $7(29 \mathrm{mg}), \mathrm{LiBr}(15 \mathrm{mg}, 0.17 \mathrm{mmol})$, and $\mathrm{Li}_{2} \mathrm{CO}_{3}(12.5 \mathrm{mg}, 0.17 \mathrm{mmol})$ in dry DMF $(0.5 \mathrm{~mL})$ was stirred at $140{ }^{\circ} \mathrm{C}$ for $2 \mathrm{~h}$ under nitrogen. The reaction mixture was cooled to room temperature, poured into dilute $\mathrm{HCl}$ and extracted with ether. The combined organic layers were washed with water, dried over $\mathrm{MgSO}_{4}$ and concentrated. The residue was purified by column chromatography (silica gel, petroleum ether-ethyl acetate, $95: 5)$ to afford compound $\mathbf{1 1}(13 \mathrm{mg}$, $60 \%$ ) as a yellow solid. mp $136-142{ }^{\circ} \mathrm{C}$ (AcOEt); $m_{\max } / \mathrm{cm}^{-1}$ $3605(\mathrm{OH}) ; d_{\mathrm{H}}(400 \mathrm{MHz}) 8.08(1 \mathrm{H}, \mathrm{dd}, J=9.2,5.6 \mathrm{~Hz}, \mathrm{CH}$ arom), $7.31(1 \mathrm{H}, \mathrm{dd}, J=10.4,2.8 \mathrm{~Hz}, \mathrm{CH}$ arom $), 7.21(1 \mathrm{H}$, ddd, $J=8.4,8.4,2.4 \mathrm{~Hz}, \mathrm{CH}$ arom $), 6.96(1 \mathrm{H}, \mathrm{d}, J=8.0 \mathrm{~Hz}$, $\mathrm{C} H$ arom), $6.53(1 \mathrm{H}, \mathrm{d}, J=8.4 \mathrm{~Hz}, \mathrm{CH}$ arom $), 5.82(1 \mathrm{H}, \mathrm{br} \mathrm{s}$, $\mathrm{OH}), 1.50\left(9 \mathrm{H}, \mathrm{s},{ }^{t} \mathrm{Bu}\right) ; d_{\mathrm{C}}(100 \mathrm{MHz}) 178.1(\mathrm{C}, \mathrm{O}-\mathrm{CO}), 162.7$ (C, $C-\mathrm{OH}), 160.3$ (C, $C$-OPiv), 149.7 (C, $C-\mathrm{F}), 128.8(2 \times C$ ), $125.5\left(\mathrm{CH},{ }^{3} J_{\mathrm{CF}}=9.0 \mathrm{~Hz}, \mathrm{CH}\right.$ arom $), 119.1(\mathrm{CH}$ arom $), 115.6$ $\left(\mathrm{CH},{ }^{2} J_{\mathrm{CF}}=25.1 \mathrm{~Hz}, \mathrm{CH}\right.$ arom $), 107.1(\mathrm{CH}$ arom $), 104.8(\mathrm{CH}$, ${ }^{2} J_{\mathrm{CF}}=23.0 \mathrm{~Hz}, \mathrm{CH}$ arom $), 77.2\left(\mathrm{C},{ }^{t} \mathrm{Bu}\right), 27.4\left(3 \times \mathrm{CH}_{3},{ }^{t} \mathrm{Bu}\right)$; $\mathrm{m} / z\left(\mathrm{CI}+\mathrm{NH}_{3}\right) 263\left(\mathrm{MH}^{+}\right), 280\left(\mathrm{MH}^{+}+\mathrm{NH}_{3}\right) ; \mathrm{m} / z$ (rel intensity) $262\left(\mathrm{M}^{+}, 15\right), 178\left(\mathrm{M}^{+}-1-\mathrm{C}_{5} \mathrm{H}_{9} \mathrm{O}, 100\right)$; HRMS calcd for $\mathrm{C}_{15} \mathrm{H}_{15} \mathrm{FO}_{3}$ : 262.100523; found 262.101006. Anal. calcd. for $\mathrm{C}_{15} \mathrm{H}_{15} \mathrm{FO}_{3}$ : C, 68.69; H, 5.76. Found: $\mathrm{C}, 68.71 ; \mathrm{H}, 6.05 \%$.

2-(7-Chloro-4-hydroxy-1-naphthyl)ethyl acetate (13). To a stirred solution of $4 \mathbf{c}(0.2 \mathrm{~g}, 0.75 \mathrm{mmol})$ in acetic acid $(7 \mathrm{~mL})$ at room temperature was added slowly pyridinium bromide perbromide $(0.24 \mathrm{~g}, 0.75 \mathrm{mmol})$. The mixture was stirred at room temperature for $15 \mathrm{~min}$, neutralised with saturated aqueous $\mathrm{Na}_{2} \mathrm{CO}_{3}$, extracted with ethyl acetate, dried and concentrated. The residue was then dissolved in DMF (4 mL). $\mathrm{Li}_{2} \mathrm{CO}_{3}(0.11 \mathrm{~g}$,
$1.5 \mathrm{mmol})$ and $\operatorname{LiBr}(0.13 \mathrm{~g}, 1.5 \mathrm{mmol})$ were added and the solution was heated for 30 minutes at $140{ }^{\circ} \mathrm{C}$, cooled to room temperature, neutralised with saturated aqueous citric acid, extracted with diethyl ether and the combined organic extracts were dried and concentrated in vacuo. The residue was purified by flash column chromatography (silica gel, petroleum etherethyl acetate, $8: 2)$ to give naphthol $\mathbf{1 3}(64 \%)$ as a brown-green solid. mp 101-104 ${ }^{\circ} \mathrm{C}\left(\mathrm{CH}_{2} \mathrm{Cl}_{2}\right.$-petroleum ether $) ; m_{\max } / \mathrm{cm}^{-1} 3358$ $(\mathrm{OH}), 1743(\mathrm{O}-C$ 回 $O) ; d_{\mathrm{H}}(400 \mathrm{MHz}) 8.23(1 \mathrm{H}, \mathrm{d}, J=8.8 \mathrm{H}$, $\mathrm{C} H$ arom $), 8.00(1 \mathrm{H}, \mathrm{d}, J=1.2 \mathrm{~Hz}, \mathrm{CH}$ arom $), 7.44(1 \mathrm{H}, \mathrm{dd}$, $J=8.8,1.6 \mathrm{~Hz}, \mathrm{C} H$ arom $), 7.19(1 \mathrm{H}, \mathrm{d}, J=7.6 \mathrm{~Hz}, \mathrm{C} H$ arom $)$, $6.75(1 \mathrm{H}, \mathrm{d}, J=7.6 \mathrm{~Hz}, \mathrm{CH}$ arom $), 6.55(1 \mathrm{H}, \mathrm{s}, \mathrm{OH}), 4.41(2 \mathrm{H}$, $\left.\mathrm{dd}, J=7.4,7.4 \mathrm{~Hz}, \mathrm{CH}_{2}-\mathrm{OAc}\right), 3.29(2 \mathrm{H}, \mathrm{dd}, J=7.2,7.2 \mathrm{~Hz}$, $\left.\mathrm{Ar}-\mathrm{CH}_{2}\right), 2.13\left(3 \mathrm{H}, \mathrm{s}, \mathrm{OC}(\mathrm{O}) \mathrm{CH}_{3}\right) ; d_{\mathrm{C}}(100 \mathrm{MHz}) 172.2(\mathrm{O}-\mathrm{CO})$, $151.2(C-\mathrm{OH}), 133.9(C-\mathrm{Cl}), 132.9\left(C-\mathrm{CH}_{2}\right), 128.2(\mathrm{CH}$ arom $)$, $125.7(\mathrm{CH}$ arom $), 123.2(C-\mathrm{C}-\mathrm{OH}), 124.6(\mathrm{CH}$ arom $), 122.7(C-$ $\left.\mathrm{C}-\mathrm{CH}_{2}\right), 122.6(\mathrm{CH}$ arom $), 108.4(\mathrm{CH}$ arom $), 64.9\left(\mathrm{CH}_{2}-\mathrm{OAc}\right)$, $31.5\left(\mathrm{Ar}-\mathrm{CH}_{2}\right), 21.1\left(\mathrm{CH}_{3}, \mathrm{OC}(\mathrm{O}) \mathrm{CH}_{3}\right) ; \mathrm{m} / \mathrm{z}\left(\mathrm{CI}+\mathrm{NH}_{3}\right) 284$ $\left(\mathrm{MH}^{+}+\mathrm{NH}_{3}\right), 282\left(\mathrm{MH}^{+}+\mathrm{NH}_{3}\right)$. Anal. calcd. for $\mathrm{C}_{14} \mathrm{H}_{13} \mathrm{ClO}_{3}$ : C, 63.52; H, 4.95. Found: C, 63.48; H, 4.92\%.

Method D. Ethyl 2-amino-7-chloronaphtho[1,2-b]furan-3carboxylate (17). To a stirred solution of tetralone $4 \mathrm{~b}(0.1 \mathrm{~g}$, $0.35 \mathrm{mmol})$ in acetic acid $(3.5 \mathrm{~mL})$ was added pyridinium bromide perbromide $(0.11 \mathrm{~g}, 0.35 \mathrm{mmol})$. The mixture was stirred at room temperature for $15 \mathrm{~min}$, neutralised with saturated aqueous $\mathrm{Na}_{2} \mathrm{CO}_{3}$, extracted with ethyl acetate, dried and concentrated. A solution of the residue in acetone $(0.7 \mathrm{~mL})$ was then added to a stirred mixture of ethyl cyanoacetate $(0.07 \mathrm{~mL}, 0.08 \mathrm{~g}$, $0.71 \mathrm{mmol})$ and $\mathrm{K}_{2} \mathrm{CO}_{3}(0.15 \mathrm{~g}, 1.07 \mathrm{mmol})$ in acetone $(0.7 \mathrm{~mL})$ at $0{ }^{\circ} \mathrm{C}$. The resulting mixture was stirred at room temperature for a further $4 \mathrm{~h}$ and then acidified with saturated aqueous citric acid, extracted with dichloromethane and the combined organic extracts were dried over $\mathrm{MgSO}_{4}$ and concentrated in vacuo. The residue was purified by flash column chromatography (silica gel, petroleum ether-ethyl acetate, $9: 1)$ to give furan $17(51 \%)$ as a brown-green solid. mp $192-195{ }^{\circ} \mathrm{C}\left(\mathrm{CH}_{2} \mathrm{Cl}_{2}-\right.$ petroleum ether); $m_{\max } / \mathrm{cm}^{-1} 3486\left(\mathrm{NH}_{2}\right), 3365\left(\mathrm{NH}_{2}\right), 1682(\mathrm{O}-\mathrm{C}$ OO $) ; d$ $(400 \mathrm{MHz}) 7.98(1 \mathrm{H}, \mathrm{d}, J=8.0 \mathrm{~Hz}, \mathrm{CH}$ arom), $7.97(1 \mathrm{H}, \mathrm{s}$, $\mathrm{C} H$ arom $), 7.91(1 \mathrm{H}, \mathrm{d}, J=8.4 \mathrm{~Hz}, \mathrm{C} H$ arom $), 7.70(1 \mathrm{H}, \mathrm{d}, J$ $=8.4 \mathrm{~Hz}, \mathrm{CH}$ arom $), 7.49(1 \mathrm{H}, \mathrm{dd}, J=8.8,1.6 \mathrm{~Hz}, \mathrm{CH}$ arom $)$, $7.30\left(2 \mathrm{H}, \mathrm{s}, \mathrm{N} H_{2}\right), 4.36\left(2 \mathrm{H}, \mathrm{ddd}, J=7.1,7.1,7.1 \mathrm{~Hz}, \mathrm{O}-\mathrm{CH}_{2}\right)$, $1.40\left(3 \mathrm{H}, \mathrm{dd}, J=7.0,7.0 \mathrm{~Hz}, \mathrm{CH}_{3}\right) ; d_{\mathrm{C}}(100 \mathrm{MHz}) 167.0(\mathrm{C}$, $\mathrm{O}-\mathrm{CO}), 166.7\left(\mathrm{C}, C-\mathrm{CO}_{2} \mathrm{Et}\right), 144.0(\mathrm{C}, C-\mathrm{OH}), 132.2(\mathrm{C}, C-\mathrm{Cl})$, $130.4\left(\mathrm{C}, \mathrm{C}-\mathrm{C}-\mathrm{CO}_{2} \mathrm{Et}\right), 128.9(\mathrm{CH}$ arom $), 128.8(\mathrm{CH}$ arom), $125.7(\mathrm{C}, \mathrm{C}-\mathrm{C}-\mathrm{OH}), 125.1(\mathrm{CH}$ arom $), 122.2(\mathrm{CH}$ arom $), 122.0$ $(\mathrm{CH}$ arom $), 120.0(\mathrm{C}, \mathrm{C}-\mathrm{CH}), 86.2\left(\mathrm{C}, C-\mathrm{NH}_{2}\right), 61.0\left(\mathrm{CH}_{2}\right.$, $\left.\mathrm{O}-\mathrm{CH}_{2}\right), 15.9\left(\mathrm{CH}_{3}\right) ; \mathrm{m} / \mathrm{z}\left(\mathrm{CI}+\mathrm{NH}_{3}\right) 292\left(\mathrm{MH}^{+}\right), 290\left(\mathrm{MH}^{+}\right)$; $\mathrm{m} / \mathrm{z}$ (rel intensity) $289\left(\mathrm{M}^{+}, 63\right), 243\left(\mathrm{M}^{+}-\mathrm{C}_{2} \mathrm{H}_{6} \mathrm{O}, 100\right)$; HRMS calcd for $\mathrm{C}_{15} \mathrm{H}_{12} \mathrm{ClNO}_{3}$ : 289.050571; found 289.046883.

Method E. 6-Chloro-2-[3-(2-hydroxyethyl)benzyl]-1-naphthol (18). To a stirred solution of tetralone $4 \mathbf{c}(0.1 \mathrm{~g}, 0.37 \mathrm{mmol})$ and benzaldehyde $(0.06 \mathrm{~mL}, 0.56 \mathrm{mmol})$ in tert-butanol $(3.7 \mathrm{~mL})$ was added potassium tert-butoxide $(0.08 \mathrm{~g}, 0.75 \mathrm{mmol})$. The resulting suspension was then refluxed for $8 \mathrm{~h}$, cooled to room temperature and acidified with saturated aqueous citric acid. The reaction mixture was extracted with ethyl acetate and the combined organic extracts were dried $\left(\mathrm{Na}_{2} \mathrm{SO}_{4}\right)$ and concentrated in vacuo. The residue was purified by flash column chromatography (silica gel, petroleum ether-ethyl acetate, $9: 1)$ to give $\mathbf{1 8}(32 \%)$ as a yellow solid. mp $140-141{ }^{\circ} \mathrm{C}\left(\mathrm{CH}_{2} \mathrm{Cl}_{2}\right.$-petroleum ether $) ; m_{\max } / \mathrm{cm}^{-1}$ $3601(\mathrm{OH}), 3530(\mathrm{OH}) ; d_{\mathrm{H}}(400 \mathrm{MHz}) 8.13(1 \mathrm{H}, \mathrm{d}, J=8.8 \mathrm{~Hz}$, $\mathrm{C} H$ arom), $7.94(1 \mathrm{H}, \mathrm{d}, J=1.6 \mathrm{~Hz}, \mathrm{CH}$ arom $), 7.41(1 \mathrm{H}, \mathrm{dd}$, $J=9.0,1.8 \mathrm{~Hz}, \mathrm{CH}$ arom), 7.25-7.34 (6H, m, $6 \times \mathrm{CH}$ arom), $4.12\left(2 \mathrm{H}, \mathrm{s}, \mathrm{CH}_{2}-\mathrm{Ph}\right), 3.95\left(2 \mathrm{H}, \mathrm{dd}, J=6.8,6.8 \mathrm{~Hz}, \mathrm{CH}_{2}-\mathrm{OH}\right)$, $3.21\left(2 \mathrm{H}, \mathrm{dd}, J=6.6,6.6 \mathrm{~Hz}, \mathrm{CH}_{2}-\mathrm{CH}_{2}-\mathrm{OH}\right) ; d_{\mathrm{C}}(100 \mathrm{MHz})$ $148.5(\mathrm{C}, C-\mathrm{OH}), 138.8(\mathrm{C}, C-\mathrm{Cl}), 133.0(\mathrm{C}, C-\mathrm{C}-\mathrm{OH}), 132.3$ (C, $\left.\mathrm{C}-\mathrm{CH}_{2}-\mathrm{Ph}\right), 131.5(\mathrm{CH}$ arom $), 129.2(2 \times \mathrm{CH}$ arom $), 128.5$ $(2 \times C \mathrm{H}$ arom $), 127.1(\mathrm{CH}$ arom $), 126.0(\mathrm{CH}$ arom $), 125.9(\mathrm{C}$, $\left.C-\mathrm{C}-\mathrm{CH}_{2}\right), 124.3(\mathrm{CH}$ arom $), 123.9\left(\mathrm{C}, \mathrm{C}-\mathrm{CH}_{2}-\mathrm{C}\right), 122.7(\mathrm{CH}$ 
arom), $119.7\left(\mathrm{C}, \mathrm{C}-\mathrm{CH}_{2}\right), 63.1\left(\mathrm{CH}_{2}, \mathrm{CH}_{2}-\mathrm{Ph}\right), 36.8\left(\mathrm{CH}_{2}\right.$, $\left.\mathrm{CH}_{2}-\mathrm{OH}\right)$, $35.6\left(\mathrm{CH}_{2}, \mathrm{CH}_{2}-\mathrm{CH}_{2}-\mathrm{OH}\right) ; \mathrm{m} / \mathrm{z}\left(\mathrm{CI}+\mathrm{NH}_{3}\right) 332$ $\left(\mathrm{MH}^{+}+\mathrm{NH}_{3}\right), 330\left(\mathrm{MH}^{+}+\mathrm{NH}_{3}\right), 315\left(\mathrm{MH}^{+}\right), 313\left(\mathrm{MH}^{+}\right)$.

Method F. N-Benzyl-6-chloro-1-naphthalenamine (19a). A solution of tetralone $\mathbf{4 b}(0.1 \mathrm{~g}, 0.35 \mathrm{mmol})$ and benzylamine $(0.08 \mathrm{~mL}, 0.71 \mathrm{mmol})$ in toluene $(3.5 \mathrm{~mL})$ was refluxed in a system equipped with a Dean-Stark apparatus. The reaction was monitored by ${ }^{1} \mathrm{H}$ NMR and after $3 \mathrm{~h}$ the starting material was completely consumed. At this point, the reaction mixture was cooled to room temperature and $\mathrm{AlCl}_{3}(0.95 \mathrm{~g}, 0.71 \mathrm{mmol})$ was added to the imine solution. The reaction was refluxed for a further $15 \mathrm{~min}$, neutralised with saturated aqueous $\mathrm{NaHCO}_{3}$ and extracted with dichloromethane. The combined organic extracts were dried over $\mathrm{Na}_{2} \mathrm{SO}_{4}$ and concentrated under reduced pressure. Flash chromatography of the residue (silica gel, petroleum ether-ethyl acetate, $98: 2$ ), gave naphthylamine $19 \mathbf{a}(75 \%)$ as a green-yellow oil. $m_{\max } / \mathrm{cm}^{-1} 3428(\mathrm{NH}) ; d_{\mathrm{H}}(400 \mathrm{MHz}) 7.80(1 \mathrm{H}, \mathrm{d}$, $J=2.0 \mathrm{~Hz}, \mathrm{C} H$ arom $), 7.75(1 \mathrm{H}, \mathrm{d}, J=8.8 \mathrm{~Hz}, \mathrm{CH}$ arom $), 7.36-$ $7.48(7 \mathrm{H}, \mathrm{m}, \mathrm{CH}$ arom), $7.18(1 \mathrm{H}, \mathrm{d}, J=8.0 \mathrm{~Hz}, \mathrm{CH}$ arom $), 6.64$ $(1 \mathrm{H}, \mathrm{d}, J=7.6 \mathrm{~Hz}, \mathrm{CH}$ arom $), 4.68(1 \mathrm{H}, \mathrm{br} \mathrm{s}, \mathrm{NH}), 4.50(2 \mathrm{H}$, s, $\left.\mathrm{CH}_{2}-\mathrm{Ph}\right) ; d_{\mathrm{C}}(100 \mathrm{MHz}) 143.4(\mathrm{C}, \mathrm{C}-\mathrm{NH}), 138.8(\mathrm{C}, \mathrm{C}-\mathrm{Cl})$, $135.2(\mathrm{C}, \mathrm{C}-\mathrm{C}-\mathrm{NH}), 131.7\left(\mathrm{C}, \mathrm{C}-\mathrm{CH}_{2}\right), 128.8(2 \times \mathrm{CH}$ arom $)$, $128.0(\mathrm{CH}$ arom $), 127.8(\mathrm{CH}$ arom $), 127.6(\mathrm{CH}$ arom $), 127.3(\mathrm{CH}$ arom), $125.4(\mathrm{CH}$ arom $), 121.9(\mathrm{CH}$ arom $), 121.6(\mathrm{C}, \mathrm{C}-\mathrm{CH})$, $116.8(2 \times \mathrm{CH}$ arom $), 105.1(\mathrm{CH}$ arom $), 48.6\left(\mathrm{CH}_{2}, \mathrm{CH}_{2}-\mathrm{Ph}\right)$; $m / z\left(\mathrm{CI}+\mathrm{NH}_{3}\right) 270\left(\mathrm{MH}^{+}+\mathrm{NH}_{3}\right), 268\left(\mathrm{MH}^{+}+\mathrm{NH}_{3}\right)$.

Methyl benzyl(6-chloro-1-naphthyl)carbamate (19b). Acetic anhydride $(0.03 \mathrm{~mL}, 0.03 \mathrm{~g}, 0.30 \mathrm{mmol})$ was added to a solution of $0.027 \mathrm{~g}(0.10 \mathrm{mmol})$ of naphthylamine 19a and DMAP ( $0.025 \mathrm{~g}, 0.20 \mathrm{mmol})$ in $\mathrm{CH}_{2} \mathrm{Cl}_{2}(1 \mathrm{~mL})$ at $0{ }^{\circ} \mathrm{C}$. The mixture was stirred for $10 \mathrm{~h}$ at room temperature and then partitioned between water and dichloromethane. The aqueous phase was extracted with dichloromethane, the combined organic extracts were dried over $\mathrm{Na}_{2} \mathrm{SO}_{4}$ and concentrated under reduced pressure. Flash chromatography of the residue (silica gel, petroleum ether-ethyl acetate, $9: 1)$ gave acetamide $19 b(97 \%)$ as a yellow oil. $m_{\max } / \mathrm{cm}^{-1} 1667(\mathrm{~N}-C$ 回O $) ; d_{\mathrm{H}}(400 \mathrm{MHz}) 7.88(1 \mathrm{H}, \mathrm{d} J$ $=2.0 \mathrm{~Hz}, \mathrm{CH}$ arom $), 7.74(1 \mathrm{H}, \mathrm{d}, J=8.0 \mathrm{~Hz}, \mathrm{CH}$ arom $), 7.66$ $(1 \mathrm{H}, \mathrm{d}, J=8.8 \mathrm{~Hz}, \mathrm{C} H$ arom $), 7.44(1 \mathrm{H}, \mathrm{dd}, J=9.0,1.8 \mathrm{~Hz}$, $\mathrm{CH}$

arom), $7.38(1 \mathrm{H}, \mathrm{dd}, J=7.8,7.8 \mathrm{~Hz}, \mathrm{CH}$ arom $), 7.22-7.23(3 \mathrm{H}$, $\mathrm{m}, 3 \times \mathrm{CH}$ arom $), 7.16-7.17(2 \mathrm{H}, \mathrm{m}, 2 \times \mathrm{CH}$ arom $), 6.96(1 \mathrm{H}, \mathrm{d}$, $J=7.2 \mathrm{~Hz}, \mathrm{C} H$ arom $), 5.55\left(1 \mathrm{H}, \mathrm{d}, J=14.0 \mathrm{~Hz}, \mathrm{CH}_{2}-\mathrm{Ph}\right), 4.32$ $\left(1 \mathrm{H}, \mathrm{d}, J=14.0 \mathrm{~Hz}, \mathrm{CH}_{2}-\mathrm{Ph}\right), 1.76\left(3 \mathrm{H}, \mathrm{s}, \mathrm{CH}_{3}\right) ; d_{\mathrm{C}}(100 \mathrm{MHz})$ $171.0(\mathrm{C}, \mathrm{N}-\mathrm{CO}), 139.0(\mathrm{C}, \mathrm{C}-\mathrm{N}), 137.5(\mathrm{C}, \mathrm{C}-\mathrm{Cl}), 135.4(\mathrm{C}$, $C-\mathrm{C}-\mathrm{N}), 132.8\left(\mathrm{C}, \mathrm{C}-\mathrm{CH}_{2}\right), 129.4(2 \times \mathrm{CH}$ arom $), 128.8(\mathrm{C}$, $C-\mathrm{CH}), 128.4(2 \times \mathrm{CH}$ arom $), 128.4(\mathrm{CH}$ arom $), 127.9(\mathrm{CH}$ arom), $127.6(\mathrm{CH}$ arom $), 127.4(\mathrm{CH}$ arom $), 127.1(\mathrm{CH}$ arom $)$, $126.9\left(\mathrm{CH}\right.$ arom), $124.3(\mathrm{CH}$ arom $), 52.6\left(\mathrm{CH}_{2}, \mathrm{CH}_{2}-\mathrm{Ph}\right), 22.3$ $\left(\mathrm{CH}_{3}\right) ; \mathrm{m} / z\left(\mathrm{CI}+\mathrm{NH}_{3}\right) 329\left(\mathrm{MH}^{+}+\mathrm{NH}_{3}\right), 327\left(\mathrm{MH}^{+}+\mathrm{NH}_{3}\right)$, $312\left(\mathrm{MH}^{+}\right), 310\left(\mathrm{MH}^{+}\right) ; \mathrm{m} / z$ (rel intensity) $309\left(\mathrm{M}^{+}, 13\right), 267$ $\left(\mathrm{M}^{+}-1-\mathrm{C}_{2} \mathrm{H}_{3} \mathrm{O}, 14\right), 91$ (100); HRMS calcd for $\mathrm{C}_{19} \mathrm{H}_{16} \mathrm{ClNO}$ : 309.092042; found 309.089706.

Method G. 3-Chloro-11H-benzo[a]carbazole (20). A mixture of tetralone $4 \mathbf{b}(0.2 \mathrm{~g}, 0.71 \mathrm{mmol})$, phenylhydrazine $(0.14 \mathrm{~mL}$, $1.4 \mathrm{mmol}$ ) and $0.5 \mathrm{~g}$ of polyphosphoric acid (PPA) was heated at $100{ }^{\circ} \mathrm{C}$ for $15 \mathrm{~min}$. The mixture was allowed to cool to room temperature and then partitioned between water and $\mathrm{CH}_{2} \mathrm{Cl}_{2}$. The aqueous phase was extracted with dichloromethane, the combined organic extracts were dried over $\mathrm{Na}_{2} \mathrm{SO}_{4}$ and concentrated under reduced pressure. Flash chromatography of the residue (silica gel, petroleum ether-ethyl acetate, $98: 2$ ) gave compound $21(56 \%)$ as an orange-brown solid. mp 212-214 ${ }^{\circ} \mathrm{C}$ (acetone-petroleum ether): $m_{\max } / \mathrm{cm}^{-1} 3604(\mathrm{NH}), 3478(\mathrm{NH})$ $d_{\mathrm{H}}(400 \mathrm{MHz}) 11.35(1 \mathrm{H}, \mathrm{s}, \mathrm{NH}), 8.49(1 \mathrm{H}, \mathrm{d}, J=8.8 \mathrm{~Hz}, \mathrm{CH}$ arom), $8.27(1 \mathrm{H}, \mathrm{d}, J=8.4 \mathrm{~Hz}, \mathrm{C} H$ arom $), 8.18(1 \mathrm{H}, \mathrm{d}, J=$ $7.6 \mathrm{~Hz}, \mathrm{C} H$ arom), $8.08(1 \mathrm{H}, \mathrm{s}, \mathrm{C} H$ arom $), 7.66(1 \mathrm{H}, \mathrm{d}, J=$ $5.6 \mathrm{~Hz}, \mathrm{C} H$ arom $), 7.64(1 \mathrm{H}, \mathrm{d}, J=6.4 \mathrm{~Hz}, \mathrm{C} H$ arom $), 7.69$ $(1 \mathrm{H}, \mathrm{d}, J=8.4 \mathrm{~Hz}, \mathrm{CH}$ arom), $7.44(1 \mathrm{H}, \mathrm{dd}, J=7.6,7.6 \mathrm{~Hz}, \mathrm{CH}$ arom), $7.28(1 \mathrm{H}, \mathrm{dd}, J=7.4,7.4 \mathrm{~Hz}, \mathrm{CH}$ arom $) ; d_{\mathrm{C}}(100 \mathrm{MHz})$ 141.0 (C), 137.0 (C), 135.1 (C), 132.1 (C), 129.3 ( $\mathrm{CH}$ arom), $127.6(\mathrm{CH}$ arom $), 126.8(\mathrm{CH}$ arom $), 125.5(C), 125.3(\mathrm{CH}$ arom), $122.6(\mathrm{CH}$ arom $), 121.6(\mathrm{CH}$ arom $), 121.5(\mathrm{CH}$ arom $)$, $120.5(\mathrm{CH}$ arom $), 120.3(\mathrm{C}), 113.2(\mathrm{CH}$ arom $) ; \mathrm{m} / z\left(\mathrm{CI}+\mathrm{NH}_{3}\right)$ $254\left(\mathrm{MH}^{+}\right), 252\left(\mathrm{MH}^{+}\right)$; Anal. calcd. for $\mathrm{C}_{16} \mathrm{H}_{10} \mathrm{NCl}: \mathrm{C}, 76.35 ; \mathrm{H}$, 4.00. Found: C, $76.54 ; \mathrm{H}, 3.98 \%$.

Method H. 4-(3-Chloro-1-propynyl)-4-hydroxy-5,8-dimethoxy1,2,3,4-tetrahydro-1-naphthalenyl pivalate (24). n-Butyllithium $(0.42 \mathrm{~mL}, 1.1 \mathrm{M}$ solution in hexanes) was added to 3-chloro-1propyne $(39 \mathrm{~mL}, 0.52 \mathrm{mmol})$ in THF $(2 \mathrm{~mL})$, which had been previously cooled to $-78^{\circ} \mathrm{C}$ under a nitrogen atmosphere. After $30 \mathrm{~min}$, tetralone $\mathbf{4 d}(145 \mathrm{mg}, 0.47 \mathrm{mmol})$ dissolved in dry THF $(1 \mathrm{ml})$ was added dropwise. The reaction was allowed to warm to room temperature over $3 \mathrm{~h}$. Saturated ammonium chloride was slowly added to the reaction followed by distilled water, and the resulting solution was extracted with ether. The organic fractions were combined, dried over $\mathrm{MgSO}_{4}$, filtered and concentrated. The residue was purified by column chromatography (silica gel, petroleum ether-ethyl acetate, $85: 15$ ) to afford compound $24(159 \mathrm{mg}, 88 \%)$ as a white solid. mp $135-138^{\circ} \mathrm{C}$ (AcOEt); $m_{\max } / \mathrm{cm}^{-1} 3537(\mathrm{OH}), 1725\left(\mathrm{O}-C\right.$ 回O); $d_{\mathrm{H}}(400 \mathrm{MHz})$ $6.93(1 \mathrm{H}, \mathrm{d}, J=8.9 \mathrm{~Hz}, \mathrm{CH}$ arom $), 6.77(1 \mathrm{H}, \mathrm{d}, J=9.0 \mathrm{~Hz}$, $\mathrm{CH}$ arom $), 6.07(1 \mathrm{H}, \mathrm{m}, 1-\mathrm{H}), 5.33(1 \mathrm{H}, \mathrm{s}, \mathrm{OH}), 4.13(2 \mathrm{H}, \mathrm{s}$, $\left.\mathrm{CH}_{2} \mathrm{Cl}\right), 3.94\left(3 \mathrm{H}, \mathrm{s}, \mathrm{OCH}_{3}\right), 3.73\left(3 \mathrm{H}, \mathrm{s}, \mathrm{OCH}_{3}\right), 2.19-2.21(2 \mathrm{H}$, m), 2.07-2.10 $(2 \mathrm{H}, \mathrm{m}), 1.15\left(9 \mathrm{H}, \mathrm{s},{ }^{t} \mathrm{Bu}\right) ; d_{\mathrm{C}}(100 \mathrm{MHz}) 177.6$ $(\mathrm{C}, C \mathrm{O}), 152.4(C), 151.0(C), 130.3(C), 123.0(C), 112.7(C \mathrm{H}$

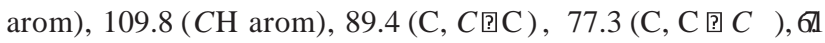
$(\mathrm{C}, \mathrm{C}-4), 63.7(\mathrm{C}, \mathrm{C}-1), 56.7\left(\mathrm{CH}_{3}, \mathrm{OCH}_{3}\right), 55.5\left(\mathrm{CH}_{3}, \mathrm{OCH}_{3}\right)$, $38.7\left(\mathrm{C},{ }^{t} \mathrm{Bu}\right), 32.0\left(\mathrm{CH}_{2}\right), 30.6\left(\mathrm{CH}_{2}\right), 27.0\left(3 \times \mathrm{CH}_{3},{ }^{t} \mathrm{Bu}\right), 25.7$ $\left(\mathrm{CH}_{2}\right) ; \mathrm{m} / \mathrm{z}\left(\mathrm{CI}+\mathrm{NH}_{3}\right) 397\left(\mathrm{M}^{+}+\mathrm{NH}_{3}\right), 395\left(\mathrm{M}^{+}+\mathrm{NH}\right)$.

5-(3-Chloro-1-propynyl)-1,4-dimethoxynaphthalene (25). To a cold $\left(0{ }^{\circ} \mathrm{C}\right)$ solution of $24(0.044 \mathrm{~g}, 0.11 \mathrm{mmol})$ in pyridine $(0.1 \mathrm{~mL})$ was added phosphorous oxychloride $(0.14 \mathrm{~mL}$, $1.54 \mathrm{mmol}$ ) over a $30 \mathrm{~min}$ period. The reaction was allowed to warm to room temperature and stirred for $40 \mathrm{~h}$. The mixture was poured into ice-water and was extracted with diethyl ether. The combined organic layers were dried over $\mathrm{MgSO}_{4}$, filtered and concentrated. The residue was purified by column chromatography (silica gel, petroleum ether-ethyl acetate, 9:1) to afford compound $25(20 \mathrm{mg}, 66 \%)$ as an amorphous solid. $m_{\max } / \mathrm{cm}^{-1} 3070$, $2931,1622,1590,1462,1408,1267,1238,1074 ; d_{\mathrm{H}}(400 \mathrm{MHz})$ $8.26(1 \mathrm{H}, \mathrm{d}, J=8.0 \mathrm{~Hz}, \mathrm{CH}$ arom $), 7.46-7.53(2 \mathrm{H}, \mathrm{m}, 2 \times \mathrm{CH}$ arom), 6.72-6.81 (2H, m, $2 \times \mathrm{CH}$ arom $), 4.45\left(2 \mathrm{H}, \mathrm{s}, \mathrm{CH} \mathrm{H}_{2} \mathrm{Cl}\right)$, $3.97\left(3 \mathrm{H}, \mathrm{s}, \mathrm{OCH}_{3}\right), 3.85\left(3 \mathrm{H}, \mathrm{s}, \mathrm{OCH}_{3}\right) ; d_{\mathrm{C}}(100 \mathrm{MHz}) 149.8$ (C, $C-\mathrm{OMe}), 148.0(\mathrm{C}, C-\mathrm{OMe}), 129.8(\mathrm{C}, \mathrm{C}-\mathrm{C}$ ? C $), 129.0(\mathrm{CH}$ arom), $125.9(C), 125.0(\mathrm{CH}$ arom $), 122.4(\mathrm{CH}$ arom $), 117.1$ $(C), 106.5(\mathrm{CH}$ arom $), 103.5(\mathrm{CH}$ arom $), 94.9(\mathrm{C}, \mathrm{C}$ Q C $), 94$ (C, $\mathrm{C}$ 回 $\mathrm{C}), 56.5\left(\mathrm{OCH}_{3}\right), 55.7\left(\mathrm{CH}_{3}, \mathrm{OCH}_{3}\right), 29.6\left(\mathrm{CH}_{2}, \mathrm{CH}_{2} \mathrm{Cl}\right)$; $\mathrm{m} / \mathrm{z}\left(\mathrm{CI}+\mathrm{NH}_{3}\right) 261\left(\mathrm{MH}^{+}\right), 279\left(\mathrm{MH}^{+}+\mathrm{NH}_{3}\right) ; \mathrm{m} / \mathrm{z}$ (rel intensity) $261\left(\mathrm{M}^{+}+1,50\right), 260\left(\mathrm{M}^{+}, 28\right), 246$ (100); HRMS calcd. for $\mathrm{C}_{15} \mathrm{H}_{13} \mathrm{ClO}_{2}$ : 260.060408; found 260.065285.

4-Hydroxy-5,8-dimethoxy-4-(phenylethynyl)-1,2,3,4-tetrahydro-1-naphthalenyl pivalate (26). $n$-Butyllithium $(0.52 \mathrm{~mL}$ $1.1 \mathrm{M}$ hexanes) was added to ethynylbenzene $(0.07 \mathrm{~mL}$, $0.64 \mathrm{mmol})$ in THF $(2.5 \mathrm{~mL})$, which had been previously cooled to $-78{ }^{\circ} \mathrm{C}$ under a nitrogen atmosphere. After $30 \mathrm{~min}$, tetralone $4 \mathbf{d}(178 \mathrm{mg}, 0.58 \mathrm{mmol})$ dissolved in dry THF $(1 \mathrm{~mL})$ was added dropwise. The reaction was allowed to warm to room temperature and stirred for $3.5 \mathrm{~h}$. Saturated ammonium chloride was slowly added to the reaction followed by distilled water, and the resulting solution, extracted with diethyl ether. The organic fractions were combined, dried over $\mathrm{MgSO}_{4}$, filtered and concentrated. The residue was purified by column chromatography (silica gel, petroleum ether-ethyl acetate, $95: 5$ ) to afford compound $26(151 \mathrm{mg}, 65 \%)$ as a white solid. $\mathrm{mp} 50-53{ }^{\circ} \mathrm{C}$ (AcOEt); $m_{\max } / \mathrm{cm}^{-1} 3540(\mathrm{OH}), 1724(\mathrm{C}$ O O $) ; d_{\mathrm{H}}(400 \mathrm{MHz}) 7.36-7.38(\mathrm{H}$ $\mathrm{m}, 2 \times \mathrm{CH}$ arom), 7.26-7.27 (3H, m, $3 \times \mathrm{CH}$ arom), $6.94(1 \mathrm{H}, \mathrm{d}$, 
$J=9.2 \mathrm{~Hz}, \mathrm{CH}$ arom $), 6.78(1 \mathrm{H}, \mathrm{d}, J=8.8 \mathrm{~Hz}, \mathrm{CH}$ arom $), 6.12$ $(1 \mathrm{H}$, br s, $1-\mathrm{H}), 5.44(1 \mathrm{H}, \mathrm{s}, \mathrm{OH}), 3.96\left(3 \mathrm{H}, \mathrm{s}, \mathrm{OCH}_{3}\right), 3.74(3 \mathrm{H}$, $\left.\mathrm{s}, \mathrm{OCH}_{3}\right), 2.15-2.31\left(4 \mathrm{H}, \mathrm{m}, 2-\mathrm{H}_{2}, 3-\mathrm{H}_{2}\right), 1.18\left(9 \mathrm{H}, \mathrm{s},{ }^{t} \mathrm{Bu}\right) ; \mathrm{d}_{\mathrm{C}}$ $(100 \mathrm{MHz}) 177.6(\mathrm{C}, \mathrm{CO}), 152.5(C), 151.1(C), 131.6(2 \times C \mathrm{H}$ arom), $131.1(C), 128.1(3 \times C H$ arom $), 122.9(C), 122.8(C)$, 112.9 ( $\mathrm{CH}$ arom), 109.6 ( $\mathrm{CH}$ arom), 92.2 (C, C-4), 82.9 (C, $C$ 回 $), 67.6(\mathrm{C}, \mathrm{C}$ 回 $\mathrm{C}), 63.9(\mathrm{CH}, \mathrm{C}-1), 56.7\left(\mathrm{CH}_{3}, \mathrm{OCH}_{3}\right)$, $\left(\mathrm{CH}_{3}, \mathrm{OCH}_{3}\right), 38.7\left(\mathrm{C},{ }^{t} \mathrm{Bu}\right), 32.4\left(\mathrm{CH}_{2}\right), 27.0\left(3 \times \mathrm{CH}_{3},{ }^{t} \mathrm{Bu}\right)$, $25.9\left(\mathrm{CH}_{2}\right) ; \mathrm{m} / z\left(\mathrm{CI}+\mathrm{NH}_{3}\right), 409\left(\mathrm{MH}^{+}\right), 305\left(\mathrm{M}^{+}-\mathrm{OPiv}\right)$.

1,4-Dimethoxy-5-(phenylethynyl)naphthalene (27). To a cold $\left(0{ }^{\circ} \mathrm{C}\right)$ solution of $\mathbf{2 6}(31 \mathrm{mg}, 0.08 \mathrm{mmol})$ in pyridine $(0.07 \mathrm{~mL})$ was added phosphorous oxychloride $(0.029 \mathrm{ml}, 0.31 \mathrm{mmol})$ over a 30 min period. The reaction was allowed to warm to room temperature and stirred for $36 \mathrm{~h}$. The mixture was poured into ice-water and was extracted with diethyl ether. The combined organic layers were dried over $\mathrm{MgSO}_{4}$, filtered and concentrated. The residue was purified by column chromatography (silica gel, petroleum ether-ethyl acetate, $9: 1$ ) to afford compound 27 (15 mg, 67\%) as an amorphous solid. $m_{\max } / \mathrm{cm}^{-1} 2934,1462$, $1407,1261,884 ; d_{\mathrm{H}}(400 \mathrm{MHz}) 8.26(1 \mathrm{H}, \mathrm{d}, J=8.4 \mathrm{~Hz}), 7.80$ $(1 \mathrm{H}, \mathrm{d}, J=7.2 \mathrm{~Hz}), 7.60-7.62(2 \mathrm{H}, \mathrm{m}), 7.45(1 \mathrm{H}, \mathrm{dd}, J=7.2$, $7.2 \mathrm{~Hz}), 7.32-7.38(3 \mathrm{H}, \mathrm{m}), 6.85(1 \mathrm{H}, \mathrm{d}, J=8.4 \mathrm{~Hz}), 6.76(1 \mathrm{H}$, $\mathrm{d}, J=8.4 \mathrm{~Hz}), 4.01(3 \mathrm{H}, \mathrm{s}), 3.98(3 \mathrm{H}, \mathrm{s}) ; d_{\mathrm{C}}(100 \mathrm{MHz}) 159.2$ $(C), 149.8(C), 133.8(C H), 131.4(C H), 128.2(3 \times C H), 127.7$ $(C \mathrm{H}), 127.0(C), 126.5(C), 125.8(C), 124.9(C H), 122.6(C H)$, 117.6 $(\mathrm{C}), 103.9(\mathrm{CH}), 91.6(2 \times \mathrm{C}), 56.9\left(\mathrm{CH}_{3}\right), 55.7\left(\mathrm{CH}_{3}\right) ; \mathrm{m} / \mathrm{z}$ $\left(\mathrm{CI}+\mathrm{NH}_{3}\right) 306\left(\mathrm{MH}^{+}+\mathrm{NH}_{3}\right), 289\left(\mathrm{MH}^{+}\right), 274\left(\mathrm{MH}^{+}+\mathrm{NH}\right)$; $\mathrm{m} / \mathrm{z}$ (rel intensity) $288\left(\mathrm{M}^{+}, 100\right), 273\left(\mathrm{M}^{+}-\mathrm{CH}_{3}, 58\right), 258\left(\mathrm{M}^{+}\right.$ $\left.-2 \times \mathrm{CH}_{3}, 19\right)$; HRMS calcd for $\mathrm{C}_{20} \mathrm{H}_{16} \mathrm{O}_{2}$ : 288.115030; found 288.113342 .

\section{Acknowledgements}

I. P.-M. thanks the Ministerio de Educación, Cultura y Deporte (Spain) for a postdoctoral fellowship and A. C.-V. thanks CONACYT (Mexico) for its generous financial support. The authors wish to thank Oya Bermek for the preparation of compound 5, and I.P.N.A., C.S.I.C. (Spain) for micromass spectra.

\section{References}

1 A. D. Martinez, J. P. Deville, J. L. Stevens and V. Behar, J. Org. Chem., 2003, 69, 991-992, and references cited therein; J. N. Kim, Y. J. Im, J. H. Gong and K. Y. Lee, Tetrahedron Lett., 2001, 42, 4195-4197, and references cited therein; A. T. Hopper, D. T. Witiak and J. Ziemniak, J. Med. Chem., 1998, 41, 420-427.
2 A. Gopalsamy, K. Lim, J. W. Ellingboe, B. Mitsner, A. Nikitenko, J. Upeslacis, T. S. Mansour, M. W. Olson, G. A. Bebernitz, D. Gringberg, B. Feld, F. J. Moy and J. O'Connell, J. Med. Chem., 2004, 47, 1893-1899; G. Bringmann, W. Saeb, M. Rückert, J. Mies, M. Michel, V. Mudogo and R. Brun, Phytochemistry, 2003, 62, 631-636, and references cited therein; A. R. Katritzky, G. Zhang and L. Xie, J. Org. Chem., 1997, 62, 721-725, and references cited therein; A. I. Meyers and J. J. Willemsen, Tetrahedron Lett., 1996, 37, 791-792; M. Medarde, A. C. Ramos, E. Caballero and J. L. Lopez, Tetrahedron Lett., 1996, 37, 2663-2666.

3 A. Cordero-Vargas, B. Quiclet-Sire and S. Z. Zard, Org. Lett., 2003, 5, 3717-3719; A. Liard, B. Quiclet-Sire, R. N. Saicic and S. Z. Zard, Tetrahedron Lett., 1997, 38, 1759-1762. For an earlier mechanistically similar approach, see B. B. Snider and L. Han, Synth. Commun., 1995, 25, 2337-2347; E. I. Heiba and R. M. Dessau, J. Am. Chem. Soc., 1972, 94, 2888-2889.

4 F. Gagosz and S. Z. Zard, Org. Lett., 2002, 4, 4345-4348.

5 G. Mehta, R. S. Senaiar and M. K. Bera, Chem. Eur. J., 2003, 9, 2264-2272.

6 For a review of benzines as dienophiles, see R. W. Hoffmann, Dehydrobenzene and Cycloalkynes, Academic Press, New York, 1967, pp. 200-239.

7 For nucleophilic substitution, see G. Murineddu, G. Cignarella, G. Chelucci, G. Loriga and G. A. Pinna, Chem. Pharm. Bull.,2002, 50, 754-759; G. A. Pinna, G. Loriga, G. Murineddu, G. Grella, M. Mura, L. Vargiu, C. Murgioni and P. L. Colla, Chem. Pharm. Bull., 2001, 49, 1406-1411.

8 J. Tsuji, Palladium Reagents and Catalysts. Innovations in Organic Synthesis, Wiley, New York, 1997.

9 For a monograph, see B. Robinson, The Fischer Indole Synthesis, Wiley, New York, 1983. For reviews, see I. I. Grandberg and V. I. Sorokin, Russ. Chem. Rev., 1974, 43, 115-128; H. J. Shine, Aromatic Rearrangements, Elsevier, New York, 1969, pp. 190-207, Ref. 489; R. J. Sundberg, The Chemistry of Indoles, Academic Press, New York, 1970, pp. 142-163; B. Robinson, Chem. Rev., 1969, 69, 227 250; Y.Lihu, Tetrahedron Lett., 2000, 41, 6981-6984.

10 P. K. Mahata, U. K. Venkatesh, S. Kumar, H. Ila and H. Junjappa, J. Org. Chem., 2003, 68, 3966-3975, and references cited therein; S. M. Barolo, A. E. Lukach and R. A. Rossi, J. Org. Chem., 2003, 68, 2807-2811 and references cited therein.

11 METHOD A: like Method A, Table 2. METHOD G: To a cold solution of substrate $(1 \mathrm{mmol})$ in pyridine $(1 \mathrm{ml})$ was added $\mathrm{POCl}_{3}$ $(15.5 \mathrm{mmol})$ and the reaction was stirred at room temperature.

12 N. P. Buu-Hoi, P. Jacquignon, N. D. Xuong and D. Lavit, J. Org. Chem., 1954, 19, 1370-1374.

13 N. Legrand, B. Quiclet-Sire and S. Z. Zard,Tetrahedron Lett., 2000, 41, 9815-9818.

14 Xanthate 1d was prepared by S. Seguin, Nouvelles réactions d'allylation radicalaires, synthése de nouveaux agents intercalants-alkylants, apparentés aux duocarmycines, par une voie radicalaire originale, PhD Thesis, 1999, Université ParisSud, pp. 158-159.

15 H. Laatsch, Liebings Ann. Chem., 1985, 12, 2420. 